\title{
Null Curve Evolution in Four-Dimensional Pseudo-Euclidean Spaces
}

\author{
José del Amor, ${ }^{1}$ Ángel Giménez, ${ }^{2}$ and Pascual Lucas ${ }^{1}$ \\ ${ }^{1}$ Departamento de Matemáticas, Universidad de Murcia, Campus de Espinardo, 30100 Murcia, Spain \\ ${ }^{2}$ Centro de Investigación Operativa, Universidad Miguel Hernández de Elche, Avenida Universidad s/n, Elche, 03202 Alicante, Spain \\ Correspondence should be addressed to Pascual Lucas; plucas@um.es
}

Received 13 April 2016; Accepted 5 May 2016

Academic Editor: Emilio Elizalde

Copyright (c) 2016 José del Amor et al. This is an open access article distributed under the Creative Commons Attribution License, which permits unrestricted use, distribution, and reproduction in any medium, provided the original work is properly cited.

\begin{abstract}
We define a Lie bracket on a certain set of local vector fields along a null curve in a 4-dimensional semi-Riemannian space form. This Lie bracket will be employed to study integrability properties of evolution equations for null curves in a pseudo-Euclidean space. In particular, a geometric recursion operator generating infinitely many local symmetries for the null localized induction equation is provided.
\end{abstract}

\section{Introduction}

Recently in $[1,2]$ a connection between the local motion of a null curve in $\mathbb{L}^{3}$ and the celebrated $\mathrm{KdV}$ equation was given. In [3] the author obtained a connection between a null curve evolution in $\mathbb{L}^{4}$ (to which we refer as the "null localized induction equation" or NLIE), and the Hirota-Satsuma coupled $\mathrm{KdV}$ (HS-cKdV) system, we briefly remind the reader of here. Hirota and Satsuma [4] proposed (perhaps up to rescaling) the HS-cKdV system

$$
\begin{aligned}
& u_{t}=\frac{1}{2} u_{x x x}+3 u u_{x}-6 v v_{x}, \\
& v_{t}=-v_{x x x}-3 u v_{x}
\end{aligned}
$$

which describes the interactions of two long waves with different dispersion relations. Many systematic methods have been employed in the literature to clarify the integrability of the HS-cKdV system: the Lax pair [5-7], the Bäcklund transformation method [8], the Darboux transformation [9$11]$, the Painleve analysis $[6,7]$, the search of infinitely many symmetries and conservation laws $[4,12,13]$, and so forth.
Fuchssteiner [12] discovered that HS-cKdV system given by (1) admits the symplectic and the cosymplectic operators:

$$
\begin{aligned}
& J(u, v)=\left(\begin{array}{cc}
\frac{1}{2} D_{x}+u D_{x}^{-1}+D_{x}^{-1} u & -2 D_{x}^{-1} v \\
-2 v D_{x}^{-1} & -2 D_{x}
\end{array}\right), \\
& \Theta(u, v) \\
& \quad=\left(\begin{array}{cc}
\frac{1}{2} D_{x}^{3}+u D_{x}+D_{x} u & D_{x} v+v D_{x} \\
D_{x} v+v D_{x} & \frac{1}{2} D_{x}^{3}+u D_{x}+D_{x} u
\end{array}\right) .
\end{aligned}
$$

Furthermore, an infinite hierarchy of symmetries for (1) was found in [13]:

$$
\begin{gathered}
\sigma_{0}=\left(u_{x}, v_{x}\right) ; \\
\sigma_{1}=\left(\frac{1}{2} u_{x x x}+3 u u_{x}-6 v v_{x},-v_{x x x}-3 u v_{x}\right) ; \\
\left\{\sigma_{2 n}=(\Theta J)^{n} \sigma_{0} ; \sigma_{2 n+1}=(\Theta J)^{n} \sigma_{1}\right\} .
\end{gathered}
$$

In this case the recursion operator $\Theta J$ is not hereditary. Hence, the bi-Hamiltonian formulation of the HS-cKdV system does not arise from a Hamiltonian pair. 
In this paper, we extend some of the results given in [1-3] to a more general background. More specifically, we generalize the Lie algebra structure defined on the local vector fields along null curves from the 3-dimensional Minkowski space to 4-dimensional semi-Riemannian space forms $M_{q}^{4}(G)$ of index $q=1$ or $q=2$ and curvature $G$. This Lie algebra together with the properties about the HS-cKdV system described above will be used to construct an infinity hierarchy of commuting symmetries for the NLIE equation in a 4dimensional pseudo-Euclidean space.

It is interesting to point out that, from a physical point of view, the 4-dimensional space is a more realistic context than the 3-dimensional background, the latter very often serving merely as a toy model. Let us recall that relativistic particles models have been described by actions defined on null curves whose Lagrangians are functions of their curvatures [14, 15]. These actions were also studied in Minkowski spaces $\mathbb{L}^{3}$ and $\mathbb{L}^{4}$ (see $[16,17]$ ), as well as in 3 -dimensional Lorentzian space forms in [18]. All these works were addressed to study variational problems on null curve spaces, and they have shown that the underlying mechanical system is governed by a stationary system of Korteweg-de Vries type. Furthermore, if $\gamma(\sigma, 0)$ is a critical point (the so-called null elastica) for the action $c \int k d \sigma$, where $c$ is a constant, then the associated solution $\gamma(\sigma, t)$ to the NLIE starting from $\gamma(\sigma, 0)$ is the null elastica evolving by rigid motions in the direction $X=$ $(1 / 2) k T+N$, where $X$ is actually the rotational Killing vector field for the null elastica (see $[16,18]$ ), and it served to determine the benchmark for the evolution equation NLIE in [1]. This idea was originally explored by Hasimoto between the elastica (an equilibrium shape of an elastic rod) and the "localized induction equation" (LIE). As might be expected, relationships with the Korteweg-de Vries evolution systems still arise when the null curve motions in 4-dimensional backgrounds are considered.

One of the many advantages of having a scalar evolution equation coming from a curve motion is that many aspects of its integrability can be elucidated from the intrinsic geometry of the involved curves (see $[19,20]$ ). Conversely, integrability properties of the curvature flow can be employed to determine integrability properties of the curve evolution equation (see $[1,21-23])$. Despite the numerous well-known connections between curve evolution equations and integrable Hamiltonian systems of PDEs, there is still a lack of understanding regarding the mechanisms and links among the different frameworks. Our overall aim here is to go further into those concerns.

The rest of this paper is organized as follows. In Section 2 we summarize some basic notions about formal variational calculus on which the Hamiltonian theory of nonlinear evolution equations is based. In Section 3 we have included some background formulas and results concerning the differential geometry of null curves in a semi-Riemannian space form. In particular, we study the properties of variation vector fields along a null curve in a semi-Riemannian space form as well as the variational formulas for its curvatures. In Section 4, the Lie bracket on the set of $\mathscr{P}$-local vector fields locally preserving the causal character along null curves in a 3-dimensional Lorentzian space given in [1] is extended to 4-dimensional
semi-Riemannian space forms. This section also includes a discussion about the connection between the geometric variational formulas for curvatures and the Hamiltonian structure for the HS-cKdV system. The above results will be employed in Section 5 to introduce the NLIE equation as a geometric realization of HS-cKdV equations and to construct a geometric recursion operator generating an infinity hierarchy of commuting symmetries for the NLIE equation.

\section{Preliminaries}

In this section we summarize some necessary notions and basic definitions from differential calculus which are relevant to the rest of the paper (see [24-26] for a very complete treatment of the subject). Let $n$ be a positive integer and consider $u_{1}, u_{2}, \ldots, u_{n}$ differentiable functions in the real variable $x$. Set

$$
u_{i}^{(m)}=\frac{d^{m} u_{i}}{d x^{m}}, \quad \text { for } m \in \mathbb{N}, i \in\{1, \ldots, n\} .
$$

Let $\mathscr{P}$ be the algebra of polynomials in $u_{1}, u_{2}, \ldots, u_{n}$ and their derivatives of arbitrary order, namely,

$$
\mathscr{P}=\mathbb{R}\left[u_{i}^{(m)}: m \in \mathbb{N}, i \in\{1, \ldots, n\}\right] .
$$

We refer to the elements of $\mathscr{P}$ whose constant term vanishes as $\mathscr{P}_{0}$. Acting on the algebra $\mathscr{P}$ is defined as derivation $\partial$ obeying:

$$
\begin{aligned}
\partial(f g) & =(\partial f) g+f(\partial g), \\
\partial\left(u_{i}^{(m)}\right) & =u_{i}^{(m+1)},
\end{aligned}
$$

thereby becoming a differential algebra.

Remark 1. In a more general setting we can consider $\mathscr{P}$, for example, to be the algebra of local functions, that is, $\mathscr{P}=$ $\bigcup_{j=1}^{\infty} \mathscr{P}_{j}$, where $\mathscr{P}_{j}$ is the algebra of locally analytic functions of $u_{1}, u_{2}, \ldots, u_{n}$ and their derivatives up to order $j$ (see [2730]). All the results and formulas established in Sections 4 and 5 involving the algebra $\mathscr{P}$ remain valid if the differential algebra of polynomials is replaced by the differential algebra of local functions. Nonetheless, the differential algebra of polynomials is sufficient for our purposes.

It is customary to take $\partial$ as the total derivative $D_{x}$ which can be viewed as

$$
D_{x}=\sum_{i=1}^{n} \sum_{m \in \mathbb{N}} u_{i}^{(m+1)} \frac{\partial}{\partial u_{i}^{(m)}} .
$$

In addition to $\partial$, other derivations $\xi$ may also be considered. The action of $\xi$ is determined if we know how $\xi$ acts on the generators of the algebra. Indeed, set

$$
a_{i, m}=\xi u_{i}^{(m)}
$$

then, for any $f \in \mathscr{P}$ we have

$$
\xi f=\sum_{i=1}^{n} \sum_{m \in \mathbb{N}} a_{i, m} \frac{\partial f}{\partial u_{i}^{(m)}} .
$$


The space of all derivations on $\mathscr{P}$, denoted by $\operatorname{der}(\mathscr{P})$, is a Lie algebra with respect to the usual commutator

$$
\left[\partial_{1}, \partial_{2}\right]=\partial_{1} \partial_{2}-\partial_{2} \partial_{1}, \quad \partial_{1}, \partial_{2} \in \operatorname{der}(\mathscr{P})
$$

Derivations commuting with the total derivative have important properties. Among others, if $[\xi, \partial]=0$, we have

$$
a_{i, m+1}=\xi u_{i}^{(m+1)}=\xi \partial u_{i}^{(m)}=\partial \xi u_{i}^{(m)}=\partial a_{i, m}
$$

Thus

$$
\xi f=\sum_{i=1}^{n} \sum_{m \in \mathbb{N}} a_{i}^{(m)} \frac{\partial f}{\partial u_{i}^{(m)}}
$$

where $a_{i}=a_{i, 0}=\xi u_{i}$. Let $A=\left(a_{1}, \ldots, a_{n}\right)$ be an element of $\mathscr{P}^{n}$ and write

$$
\partial_{A}=\sum_{i=1}^{n} \sum_{m \in \mathbb{N}} a_{i}^{(m)} \frac{\partial}{\partial u_{i}^{(m)}}
$$

The set of derivations $\partial_{A}$ is a Lie subalgebra of $\operatorname{der}(\mathscr{P})$, and it induces a Lie algebra on the space $\mathscr{P}^{n}$. Indeed, a direct computation shows that $\partial_{A}$ are derivations in $\mathscr{P}$ verifying $\left[\partial_{A}, \partial_{B}\right]=\partial_{[A, B]}$, where $[A, B]=\partial_{A} B-\partial_{B} A$; that is,

$$
[A, B]_{j}=\sum_{i=1}^{n} \sum_{m \in \mathbb{N}}\left(a_{i}^{(m)} \frac{\partial b_{j}}{\partial u_{i}^{(m)}}-b_{i}^{(m)} \frac{\partial a_{j}}{\partial u_{i}^{(m)}}\right) .
$$

This latter commutator can also be expressed with the aid of Fréchet derivatives as $[A, B]=B^{\prime}[A]-A^{\prime}[B]$, where

$$
A^{\prime}[B]=\left.\frac{d}{d t}\right|_{t=0} \partial_{A}(u+t B), \quad u=\left(u_{1}, u_{2}, \ldots, u_{n}\right) .
$$

We will refer to $\partial_{A}$ as an evolution derivation (or a vector field, provided that no confusion is possible), and the algebra of all evolution derivations will be denoted by $\operatorname{der}^{*}(\mathscr{P})$. Observe that, in particular, if we take $A=u^{\prime}=\left(u_{1}^{\prime}, \ldots, u_{n}^{\prime}\right)$, then $\partial=$ $\partial_{u^{\prime}}$, with $u^{\prime}=D_{x} u$.

Set $u^{(m)}=\left(u_{1}^{(m)}, u_{2}^{(m)}, \ldots, u_{n}^{(m)}\right)$ for $m \in \mathbb{N}$. Consider an evolution equation of the form

$$
\frac{\partial u}{\partial t}=F\left(u, u^{(1)}, u^{(2)}, \ldots\right)
$$

where $F=\left(f_{1}, \ldots, f_{n}\right)$ is an element of $\mathscr{P}^{n}$. An element $S=\left(s_{1}, s_{2}, \ldots, s_{n}\right) \in \mathscr{P}^{n}$ is called a symmetry of the evolution equation (16) if and only if $[F, S]=F^{\prime}[S]-S^{\prime}[F]=0$. Symmetries of integrable equations can often be generated by recursion operators which are linear operators mapping a symmetry to a new symmetry. A linear differential operator $\mathscr{R}: \mathscr{P}^{n} \rightarrow \mathscr{P}^{n}$ is a recursion operator for the evolution equation (16) if it is invariant under $F$, that is, $L_{F} \mathscr{R}=0$, where $L_{F}$ is the Lie derivative acting as $L_{F} A=[F, A]$ for all $A \in \mathscr{P}^{n} . \mathscr{R}$ is said to be hereditary if for an arbitrary vector field $F \in \mathscr{P}^{n}$ the relation $L_{\mathscr{R} F} \mathscr{R}=\mathscr{R} L_{F} \mathscr{R}$ is verified.

\section{Null Curve Variations in $M_{q}^{4}(G)$}

The geometry of null curves is quite different from the nonnull ones, so let us review the relevant results, going further into what concerns us most for later work.

A semi-Riemannian manifold $\left(M_{q}^{n}, g\right)$ is an $n$-dimensional differentiable manifold $M_{q}^{n}$ endowed with a nondegenerate metric tensor $g$ with signature $(n-q, q)$. The metric tensor $g$ will be also denoted by $\langle\cdot, \cdot\rangle$ and the Levi-Civita connection by $\nabla$. The sectional curvature of a nondegenerate plane generated by $\{u, v\}$ is

$$
K(u, v)=\frac{\langle R(u, v) u, v\rangle}{\langle u, u\rangle\langle v, v\rangle-\langle u, v\rangle^{2}},
$$

where $R$ is the semi-Riemannian curvature tensor given by

$$
R(X, Y) Z=-\nabla_{X} \nabla_{Y} Z+\nabla_{Y} \nabla_{X} Z+\nabla_{[X, Y]} Z .
$$

Semi-Riemannian manifolds with constant sectional curvature are called semi-Riemannian space forms. It is a wellknown fact that the curvature tensor $R$ adopts a simple formula in these manifolds:

$$
R(X, Y) Z=G\{\langle Z, X\rangle Y-\langle Z, Y\rangle X\},
$$

where $G$ is the constant sectional curvature. When the curvature $G$ vanishes, then $M_{q}^{n}$ is called pseudo-Euclidean space and will be denoted by $\mathbb{R}_{q}^{n}$.

Let $M_{q}^{4}(G)$ denote a 4-dimensional semi-Riemannian space form with index $q=1,2$, background gravitational field $\langle\cdot, \cdot\rangle$, and Levi-Civita connection $\nabla$. A tangent vector $v$ is time-like if $\langle v, v\rangle\langle 0$, space-like if $\langle v, v\rangle>0$ or $v=0$, and null if $\langle v, v\rangle=0$. Therefore, a parametrized curve $\gamma: I \rightarrow M_{q}^{4}(G)$ is called null if its tangent vector is null at all points in the curve. Fixing a constant $a>0$, we can consider (if $\gamma$ is not a geodesic) the parameter $\sigma_{a}$ given by

$$
\sigma_{a}(s)=\frac{1}{\sqrt{a}} \int\left\langle\nabla_{\gamma^{\prime}(s)} \gamma^{\prime}(s), \nabla_{\gamma^{\prime}(s)} \gamma^{\prime}(s)\right\rangle^{1 / 4} d s,
$$

where $s$ is any parameter. When $a=1$ this parameter agrees with the pseudo arc-length parameter $\sigma$ for the null curve. In fact, it is easy to show that $\sigma_{a}$ is nothing but a linear reparametrization of the pseudo arc-length parameter and it verifies

$$
\left\langle\nabla_{\gamma^{\prime}\left(\sigma_{a}\right)} \gamma^{\prime}\left(\sigma_{a}\right), \nabla_{\gamma^{\prime}\left(\sigma_{a}\right)} \gamma^{\prime}\left(\sigma_{a}\right)\right\rangle=a^{2} .
$$

Throughout this paper it will be supposed that we have fixed a constant $a, \sigma_{a}$ will be denoted by $\sigma$, and we will also refer to it as the pseudo arc-length parameter. The Cartan frame of a nongeodesic null curve $\gamma: I \rightarrow M_{q}^{4}(G)$, verifying that $\left\{\gamma^{\prime}(\sigma), \gamma^{\prime \prime}(\sigma), \gamma^{\prime \prime \prime}(\sigma)\right\}$ is linearly independent for all $\sigma \in I$, is given by $\left\{T=\gamma^{\prime}(\sigma), W_{1}, N, W_{2}\right\}$, where

$$
\begin{aligned}
\langle T, T\rangle & =\langle N, N\rangle=0, \\
\langle T, N\rangle & =-1, \\
\left\langle W_{i}, T\right\rangle & =\left\langle W_{i}, N\right\rangle=0, \\
\left\langle W_{i}, W_{i}\right\rangle & =\varepsilon_{i},
\end{aligned}
$$


with $i=1,2$. The Cartan equations read

$$
\begin{aligned}
\nabla_{T} T & =a W_{1}, \\
\nabla_{T} W_{1} & =-k_{1} T+a \varepsilon_{1} N, \\
\nabla_{T} N & =-\varepsilon_{1} k_{1} W_{1}+\varepsilon_{2} k_{2} W_{2}, \\
\nabla_{T} W_{2} & =k_{2} T,
\end{aligned}
$$

where $\nabla_{T}$ denotes the covariant derivative along $\gamma$ and $k_{1}$, $k_{2}$ are the curvatures of the curve. The fundamental theorem for null curves tells us that $k_{1}$ and $k_{2}$ determine completely the null curve up to semi-Riemannian isometries (see [31]). Even more, if functions $k_{1}$ and $k_{2}$ are given we can always construct a null curve, pseudo arc-length parametrized, whose curvature functions are precisely $k_{1}$ and $k_{2}$. Then any local scalar geometrical invariant defined along a null curve can always be expressed as a function of its curvatures and derivatives. A nongeodesic null curve being pseudo arclength parametrized and admitting a Cartan frame as above is called a Cartan curve. The bundle given by $\operatorname{span}\left\{W_{1}, W_{2}\right\}$ is known as the screen bundle of $\gamma$ (see [31]). Projections of the variation vector fields onto the screen bundle will play a leading role in this research.

Let $\gamma$ be a null curve, for the sake of simplicity the letter $\gamma$ will also denote a variation of null curves (null variation) $\gamma=$ $\gamma(s, t): I \times(-\zeta, \zeta) \rightarrow M_{q}^{4}(G)$ with $\gamma(s, 0)$ the initial null curve. Associated with such a variation is the variation vector field $V(s)=V(s, 0)$, where $V=V(s, t)=(\partial \gamma / \partial t)(s, t)$. We denote by $\eta$ the differentiable function verifying $(\partial \gamma / \partial s)(s, t)=$ $\eta(s, t) T(s, t)$ and by $D / \partial t$ the covariant derivative along the curves $\gamma_{s}(t)=\gamma(s, t)$. We write $\gamma(\sigma, t), k_{i}(\sigma, t), V(\sigma, t)$, and so forth, for the corresponding objects in the pseudo arc-length parametrization.

Definition 2. Let $\mathfrak{X}(\gamma)$ be the set of smooth vector fields along $\gamma$. We say that $V \in \mathfrak{X}(\gamma)$ locally preserves the causal character if $\left\langle\nabla_{T} V, T\right\rangle=0$. We also say that $V$ locally preserves the pseudo arc-length parameter along $\gamma$ if $\eta(s, t)$ satisfies $\left.(\partial \eta / \partial t)\right|_{t=0}=0$.

The following properties for null variations can be found in [17] when $a=\varepsilon_{1}=\varepsilon_{2}=1$, but they can be easily adapted to the general situation.

Lemma 3. If $\gamma$ is a null variation, then its variation vector field $\checkmark$ verifies

$$
\begin{aligned}
\left\langle\nabla_{T} V, T\right\rangle & =0, \\
\left.\frac{\partial \eta}{\partial t}\right|_{t=0} & =-\frac{1}{2 a} \rho_{V} \eta, \\
{[V, T] } & =\frac{1}{2 a} \rho_{V} T,
\end{aligned}
$$

where $\rho_{V}=-\varepsilon_{1}\left\langle\nabla_{T}^{2} V, W_{1}\right\rangle$.

Thus we obtain that $V$ locally preserves the causal character and, moreover, $V$ locally preserves the pseudo arc-length parameter if and only if $\rho_{V}=0$, which in such a case also entails commutation of $T$ and $V$. We define some functions that will play a key role in the rest of the paper, namely, given a vector field $V \in \mathfrak{X}(\gamma)$ we consider the following projections of $V$ and $\nabla_{T} V$ on the screen bundle given by

$$
\begin{aligned}
h_{V} & :=\varepsilon_{1}\left\langle V, W_{1}\right\rangle, \\
l_{V} & :=\varepsilon_{2}\left\langle V, W_{2}\right\rangle, \\
\varphi_{V} & :=\varepsilon_{1}\left\langle\nabla_{T} V, W_{1}\right\rangle, \\
\psi_{V} & :=\varepsilon_{2}\left\langle\nabla_{T} V, W_{2}\right\rangle .
\end{aligned}
$$

Lemma 4. With the above notation, the following assertions hold:

(a) $\left.(D T / \partial t)\right|_{t=0}=-\alpha_{V} T+\varphi_{V} W_{1}+\psi_{V} W_{2}$;

(b) $\left.\left(D W_{1} / \partial t\right)\right|_{t=0}=-\beta_{V} T+\varepsilon_{1} \varphi_{V} N+(1 / a) \psi_{V}^{\prime} W_{2}$,

(c) $\left.(D N / \partial t)\right|_{t=0}=-\varepsilon_{1} \beta_{V} W_{1}+\alpha_{V} N+\left(\varepsilon_{1} / a\right) \delta_{V} W_{2}$,

(d) $\left.\left(D W_{2} / \partial t\right)\right|_{t=0}=\left(\varepsilon_{1} \varepsilon_{2} / a\right) \delta_{V} T-\left(\varepsilon_{1} \varepsilon_{2} / a\right) \psi_{V}^{\prime} W_{1}+\varepsilon_{2} \psi_{V} N$,

(e) $\left.\left(\partial k_{1} / \partial t\right)\right|_{t=0}=\left(1 / a^{2}\right) \varphi_{V}^{\prime \prime \prime}+(1 / a)\left[\left(k_{1} \varphi_{V}\right)^{\prime}+k_{1} \varphi_{V}^{\prime}\right]-$ $(1 / a)\left[\left(k_{2} \psi_{V}\right)^{\prime}+k_{2} \psi_{V}^{\prime}\right]+(1 / a)\left[(1 / 2 a) \rho_{V}^{\prime \prime}+k_{1} \rho_{V}-2 G g_{V}^{\prime}\right]$,

(f) $\left.\left(\partial k_{2} / \partial t\right)\right|_{t=0}=\left(\varepsilon_{1} \varepsilon_{2} / a^{2}\right) \psi_{V}^{\prime \prime \prime}+\left(\varepsilon_{1} \varepsilon_{2} / a\right)\left[\left(k_{1} \psi_{V}\right)^{\prime}+\right.$ $\left.k_{1} \psi_{V}^{\prime}\right]+(1 / a)\left[\left(k_{2} \varphi_{V}\right)^{\prime}+k_{2} \varphi_{V}^{\prime}\right]+(1 / a) k_{2} \rho_{V}-\varepsilon_{2} G l_{V}$,

where

$$
\begin{aligned}
& \alpha_{V}=\frac{1}{a}\left(\varphi_{V}^{\prime}+\frac{1}{2} \rho_{V}\right), \\
& \beta_{V}=\frac{1}{a}\left(\alpha_{V}^{\prime}+k_{1} \varphi_{V}-k_{2} \psi_{V}-G g_{V}\right), \\
& \delta_{V}=\frac{1}{a} \psi_{V}^{\prime \prime}+k_{1} \psi_{V}+\varepsilon_{1} \varepsilon_{2} k_{2} \varphi_{V} .
\end{aligned}
$$

Proof. Set $\nabla_{V}=D / \partial t$ the covariant derivative. From (24) we obtain

$$
\begin{aligned}
\nabla_{V} T= & -\left\langle\nabla_{V} T, N\right\rangle T+\varepsilon_{1}\left\langle\nabla_{V} T, W_{1}\right\rangle W_{1} \\
& +\varepsilon_{2}\left\langle\nabla_{V} T, W_{2}\right\rangle W_{2} \\
= & -\left\langle\nabla_{T} V, N\right\rangle T+\frac{1}{2 a} \rho_{V} T+\varphi_{V} W_{1}+\psi_{V} W_{2} \\
= & -\frac{1}{a}\left(\varphi_{V}^{\prime}+\frac{1}{2} \rho_{V}\right) T+\varphi_{V} W_{1}+\psi_{V} W_{2} \\
= & -\alpha_{V} T+\varphi_{V} W_{1}+\psi_{V} W_{2},
\end{aligned}
$$


where $\left\langle\nabla_{T} V, N\right\rangle=(1 / a)\left(\varphi_{V}^{\prime}+\rho_{V}\right)$ has been used. Now, taking into account formulas (18), (19), and (27) we have

$$
\begin{aligned}
& \nabla_{V} W_{1}=\frac{1}{a} \nabla_{V} \nabla_{T} T=\frac{1}{a}\left(\nabla_{T} \nabla_{V} T+\nabla_{[V, T]} T\right. \\
& -R(V, T) T)=\frac{1}{a}\left[\nabla_{T}\left(-\alpha_{V} T+\varphi_{V} W_{1}+\psi_{V} W_{2}\right)\right. \\
& \left.+\frac{1}{2 a} \rho_{V} \nabla_{T} T+G g_{V} T\right] \\
& =\frac{1}{a}\left[\left(-\alpha_{V}^{\prime}-k_{1} \varphi_{V}+k_{2} \psi+G g_{V}\right) T+a \varepsilon_{1} \varphi_{V} N\right. \\
& \left.+\psi_{V}^{\prime} W_{2}\right]=-\beta_{V} T+\varepsilon_{1} \varphi_{V} N+\frac{1}{a} \psi_{V}^{\prime} W_{2} .
\end{aligned}
$$

Considering again (18), (19), (27), and (28) we deduce

$$
\begin{aligned}
& a \varepsilon_{1} \nabla_{V} N=\nabla_{V} \nabla_{T} W_{1}+\nabla_{V}\left(k_{1} T\right)=\nabla_{T} \nabla_{V} W_{1} \\
& +\nabla_{[V, T]} W_{1}-R(V, T) W_{1}+V\left(k_{1}\right) T+k_{1} \nabla_{V} T \\
& =\left(-\beta_{V}^{\prime}+\frac{1}{a} k_{2} \psi_{V}^{\prime}-\frac{1}{2 a} k_{1} \rho_{V}-k_{1} \alpha_{V}+V\left(k_{1}\right)\right. \\
& \left.+\frac{G}{a} g_{V}^{\prime}\right) T-a \beta_{V} W_{1}+a \varepsilon_{1} \alpha_{V} N+\delta_{V} W_{2} .
\end{aligned}
$$

Since $\left\langle\nabla_{V} N, N\right\rangle=0$, the tangent component of $\nabla_{V} N$ vanishes and the expression for $V\left(k_{1}\right)$ becomes

$$
\begin{aligned}
V\left(k_{1}\right)= & \frac{1}{a^{2}} \varphi_{V}^{\prime \prime \prime}+\frac{1}{a}\left[\left(k_{1} \varphi_{V}\right)^{\prime}+k_{1} \varphi_{V}^{\prime}\right] \\
& -\frac{1}{a}\left[\left(k_{2} \psi_{V}\right)^{\prime}+k_{2} \psi_{V}^{\prime}\right] \\
& +\frac{1}{a}\left[\frac{1}{2 a} \rho_{V}^{\prime \prime}+k_{1} \rho_{V}-2 G g_{V}^{\prime}\right] .
\end{aligned}
$$

As a consequence the vector field $\nabla_{V} N$ boils down to

$$
\nabla_{V} N=-\varepsilon_{1} \beta_{V} W_{1}+\alpha_{V} N+\frac{\varepsilon_{1}}{a} \delta_{V} W_{2} .
$$

Finally, a similar computation leads to

$$
\begin{aligned}
& \varepsilon_{2} k_{2} \nabla_{V} W_{2}=\nabla_{V} \nabla_{T} N+\varepsilon_{1} V\left(k_{1}\right) W_{1}+\varepsilon_{1} k_{1} \nabla_{V}\left(W_{1}\right) \\
& -\varepsilon_{2} V\left(k_{2}\right) W_{2}=\frac{\varepsilon_{1}}{a} k_{2} \delta_{V} T+\left(\varepsilon_{1} V\left(k_{1}\right)-\varepsilon_{1} \beta_{V}^{\prime}\right. \\
& \left.-\varepsilon_{1} \frac{k_{1}}{a}\left(\varphi_{V}^{\prime}+\rho_{V}\right)+\frac{\varepsilon_{1}}{a} G g_{V}^{\prime}\right) W_{1}+\left(\alpha_{V}^{\prime}-a \beta_{V}\right. \\
& \left.+k_{1} \varphi_{V}-G g_{V}\right) N+\left(\frac{\varepsilon_{2}}{a} k_{2}\left(\varphi_{V}^{\prime}+\rho_{V}\right)+\frac{\varepsilon_{1}}{a} \delta_{V}^{\prime}\right. \\
& \left.+\frac{\varepsilon_{1}}{a} k_{1} \psi_{V}^{\prime}-\varepsilon_{2} V\left(k_{2}\right)-G l_{V}\right) W_{2} .
\end{aligned}
$$

In the same way, since the component of $W_{2}$ in $\nabla_{V} W_{2}$ vanishes, we deduce

$$
\begin{aligned}
V\left(k_{2}\right)= & \frac{\varepsilon_{1} \varepsilon_{2}}{a^{2}} \psi_{V}^{\prime \prime \prime}+\frac{\varepsilon_{1} \varepsilon_{2}}{a}\left[\left(k_{1} \psi_{V}\right)^{\prime}+k_{1} \psi_{V}^{\prime}\right] \\
& +\frac{1}{a}\left[\left(k_{2} \varphi_{V}\right)^{\prime}+k_{2} \varphi_{V}^{\prime}\right]+\frac{1}{a} k_{2} \rho_{V}-\varepsilon_{2} G l_{V}, \\
\nabla_{V} W_{2}= & \frac{\varepsilon_{1} \varepsilon_{2}}{a} \delta_{V} T-\frac{\varepsilon_{1} \varepsilon_{2}}{a} \psi_{V}^{\prime} W_{1}+\varepsilon_{2} \psi_{V} N
\end{aligned}
$$

Consider $\Lambda$ the space of pseudo arc-length parametrized null curves in $M_{q}^{4}(G)$. For $\gamma \in \Lambda$, it is easy to see that $T_{\gamma} \Lambda$ is the set of all vector fields associated with variations of pseudo arc-length parametrized null curves starting from $\gamma$. It is clear that a vector field in $T_{\gamma} \Lambda$ locally preserves the causal character and the pseudo arc-length parameter. The converse can also be proved applying a similar procedure as in [2].

Proposition 5. A vector field $V$ along $\gamma \in \Lambda$ is tangent to $\Lambda$ if and only if it locally preserves the causal character and the pseudo arc-length parameter; that is,

$$
T_{\gamma} \Lambda=\left\{V \in \mathfrak{X}(\gamma):\left\langle\nabla_{T} V, T\right\rangle=\left\langle\nabla_{T}^{2} V, W_{1}\right\rangle=0\right\} .
$$

Consequently, if $V$ is expressed by $V=f_{V} T+h_{V} W_{1}+g_{V} N+$ $l_{V} W_{2}$, where $f_{V}, h_{V}, g_{V}$, and $l_{V}$ are smooth functions, then $V \in$ $T_{\gamma} \Lambda$ if and only if

$$
\begin{aligned}
& f_{V} \\
& \quad=-\frac{1}{2 a}\left[h_{V}^{\prime}+a k_{1} D_{\sigma}^{-1}\left(h_{V}\right)-a D_{\sigma}^{-1}\left(k_{1} h_{V}-k_{2} l_{V}\right)\right], \\
& g_{V}=-\varepsilon_{1} a D_{\sigma}^{-1}\left(h_{V}\right),
\end{aligned}
$$

where $D_{\sigma}^{-1}$ is a formal indefinite $\sigma$-integral. Furthermore,

$$
\begin{aligned}
& \left(\begin{array}{c}
\varphi_{V} \\
\psi_{V}
\end{array}\right) \\
& \quad=\left(\begin{array}{cc}
\frac{a}{2}\left(\frac{1}{a} D_{\sigma}+k_{1} D_{\sigma}^{-1}+D_{\sigma}^{-1} k_{1}\right) & -\frac{a}{2} D_{\sigma}^{-1} k_{2} \\
-\varepsilon_{1} \varepsilon_{2} a k_{2} D_{\sigma}^{-1} & D_{\sigma}
\end{array}\right)
\end{aligned}
$$

$$
\cdot\left(\begin{array}{l}
h_{V} \\
l_{V}
\end{array}\right) \text {. }
$$

Proof. For a generic vector field $V$ we obtain

$$
\begin{aligned}
& \nabla_{T} V=\left(f_{V}^{\prime}-k_{1} h_{V}+k_{2} l_{V}\right) T+\left(a f_{V}+h_{V}^{\prime}-\varepsilon_{1} k_{1} g_{V}\right) \\
& \cdot W_{1}+\left(\varepsilon_{1} a h_{V}+g_{V}^{\prime}\right) N+\left(l_{V}^{\prime}+\varepsilon_{2} k_{2} g_{V}\right) W_{2} ; \\
& \nabla_{T}^{2} V=\left[\left(f_{V}^{\prime}-k_{1} h_{V}+k_{2} l_{V}\right)^{\prime}\right. \\
& \left.\quad-k_{1}\left(a f_{V}+h_{V}^{\prime}-\varepsilon_{1} k_{1} g_{V}\right)+k_{2}\left(l_{V}^{\prime}+\varepsilon_{2} k_{2} g_{V}\right)\right] T
\end{aligned}
$$




$$
\begin{aligned}
& +\left[a\left(f_{V}^{\prime}-k_{1} h_{V}+k_{2} l_{V}\right)+\left(a f_{V}+h_{V}^{\prime}-\varepsilon_{1} k_{1} g_{V}\right)^{\prime}\right. \\
& \left.-\varepsilon_{1} k_{1}\left(\varepsilon_{1} a h_{V}+g_{V}^{\prime}\right)\right] W_{1} \\
& +\left[a \varepsilon_{1}\left(a f_{V}+h_{V}^{\prime}-\varepsilon_{1} k_{1} g_{V}\right)+\left(\varepsilon_{1} a h_{V}+g_{V}^{\prime}\right)^{\prime}\right] N \\
& +\left[\varepsilon_{2} k_{2}\left(\varepsilon_{1} a h_{V}+g_{V}^{\prime}\right)+\left(l_{V}^{\prime}+\varepsilon_{2} k_{2} g_{V}\right)^{\prime}\right] W_{2} .
\end{aligned}
$$

If $V \in T_{\gamma} \Lambda$, Lemma 3 implies that

$$
\left\langle\nabla_{T} V, T\right\rangle=\left\langle\nabla_{T}^{2} V, W_{1}\right\rangle=0 .
$$

In such a case, by using (37) and (38), we deduce that

$$
\begin{array}{r}
\varepsilon_{1} a h_{V}+g_{V}^{\prime}=0, \\
2 a f_{V}^{\prime}+h_{V}^{\prime \prime}-\varepsilon_{1}\left(k_{1} g_{V}\right)^{\prime}-a k_{1} h_{V}+a k_{2} l_{V}=0 .
\end{array}
$$

Last equations easily give rise to (35). Expression (35) becomes

$$
\begin{aligned}
f_{V}= & -\frac{1}{2 a} h_{V}^{\prime}-\frac{1}{2} k_{1} D_{\sigma}^{-1}\left(h_{V}\right)+\frac{1}{2} D_{\sigma}^{-1}\left(k_{1} h_{V}\right) \\
& -\frac{1}{2} D_{\sigma}^{-1}\left(k_{2} l_{V}\right),
\end{aligned}
$$

and the following holds:

$$
\begin{aligned}
& \varphi_{V}=\varepsilon_{1}\left\langle\nabla_{T} V, W_{1}\right\rangle=a f_{V}+h_{V}^{\prime}-\varepsilon_{1} k_{1} g_{V}, \\
& \psi_{V}=\varepsilon_{2}\left\langle\nabla_{T} V, W_{2}\right\rangle=l_{V}^{\prime}+\varepsilon_{2} k_{2} g_{V} .
\end{aligned}
$$

Replacing $f_{V}$ into (42) and rearranging terms, we easily obtain (36). Conversely, if $V$ is a vector field verifying (39), then it arises from an infinitesimal variation of null curves. Indeed, according to the Cartan equations (23), Lemma 4, and formulas (35) and (42), we consider the matrices

$$
\begin{aligned}
K_{\gamma} & =\left(\begin{array}{ccccc}
0 & 0 & 0 & G & 0 \\
1 & 0 & -k_{1} & 0 & k_{2} \\
0 & a & 0 & -\varepsilon_{1} k_{1} & 0 \\
0 & 0 & \varepsilon_{1} a & 0 & 0 \\
0 & 0 & 0 & \varepsilon_{2} k_{2} & 0
\end{array}\right), \\
P & =\left(\begin{array}{ccccc}
0 & G g_{V} & -\varepsilon_{1} G h_{V} & G f_{V} & -\varepsilon_{2} G l_{V} \\
f_{V} & -\alpha_{V} & -\beta_{V} & 0 & \frac{1}{a} \varepsilon_{1} \varepsilon_{2} \delta_{V} \\
h_{V} & \varphi_{V} & 0 & -\varepsilon_{1} \beta_{V} & -\frac{\varepsilon_{1} \varepsilon_{2}}{a} \psi_{V}^{\prime} \\
g_{V} & 0 & \varepsilon_{1} \varphi_{V} & \alpha_{V} & \varepsilon_{2} \psi_{V} \\
l_{V} & \psi_{V} & \frac{1}{a} \psi_{V}^{\prime} & \frac{\varepsilon_{1}}{a} \delta_{V} & 0
\end{array}\right),
\end{aligned}
$$

verifying

$$
\begin{aligned}
& \frac{\partial F}{\partial \sigma}=F \cdot K_{\gamma}, \\
& \frac{\partial P}{\partial \sigma}=C-\left[K_{\gamma}, P\right],
\end{aligned}
$$

where

$$
\begin{aligned}
& F=\left(\begin{array}{ccccc}
\gamma & T & W_{1} & N & W_{2}
\end{array}\right), \\
& C=\left(\begin{array}{ccccc}
0 & 0 & 0 & 0 & 0 \\
0 & 0 & c_{1} & 0 & c_{2} \\
0 & 0 & 0 & -\varepsilon_{1} c_{1} & 0 \\
0 & 0 & 0 & 0 & 0 \\
0 & 0 & 0 & \varepsilon_{2} c_{2} & 0
\end{array}\right),
\end{aligned}
$$

where the functions $c_{1}$ and $c_{2}$ are given by formulas (e) and (f) given in Lemma 4, respectively, with $\rho_{V}=0$. Following the same procedure as described in Lemma 1 of [2] we can construct a null curve variation of $\gamma$ whose variation vector field is $V$.

From Proposition 5, a tangent vector field $V \in T_{\gamma} \Lambda$ and its covariant derivative $\nabla_{T} V$ are expressed by

$$
\begin{aligned}
& \text { V } \\
& \begin{aligned}
= & -\frac{1}{2 a}\left[h_{V}^{\prime}+a k_{1} D_{\sigma}^{-1}\left(h_{V}\right)-a D_{\sigma}^{-1}\left(k_{1} h_{V}-k_{2} l_{V}\right)\right] T \\
& +h_{V} W_{1}-\varepsilon_{1} a D_{\sigma}^{-1}\left(h_{V}\right) N+l_{V} W_{2},
\end{aligned} \\
& \nabla_{T} V=-\frac{1}{a} \varphi_{V}^{\prime} T+\varphi_{V} W_{1}+\psi_{V} W_{2} .
\end{aligned}
$$

Remark 6. Observe that a tangent vector field $V \in T_{\gamma} \Lambda$ is completely determined by the differentiable functions $h_{V}$ and $l_{V}$ and two constants, since the operator $D_{\sigma}^{-1}$ is used twice; once for obtaining $g_{V}$ from $h_{V}$ and once more for obtaining $f_{V}$ from $h_{V}$ and $l_{V}$. Therefore, given two differentiable functions $h_{V}$ and $l_{V}$ and two constants, we can construct a vector field locally preserving pseudo arc-length parameter along $\gamma$ whose projections on the screen bundle are precisely $h_{V}$ and $l_{V}$. Both constants could be determined or related if constraints on null curve variation are considered, but, for our algebraic purposes, we will consider generic constants.

\section{A Lie Algebra Structure on Local Vector Fields}

Our objective now is to define a Lie algebra structure on the set of local vector fields which locally preserve the causal character. To this end, we need first to set up the spaces in which we are going to work. Let $k_{1}$ and $k_{2}$ be smooth functions defined on an interval $I$ and set $\mathscr{P}$ the real algebra of polynomials in $k_{1}, k_{2}$ and their derivatives of arbitrary order; that is,

$$
\mathscr{P}=\mathbb{R}\left[k_{1}^{(m)}, k_{2}^{(n)}:(m, n) \in \mathbb{N}^{2}\right],
$$

where $k_{1}^{(m)}=k_{1}^{(m)}(\sigma)$ and $k_{2}^{(n)}=k_{2}^{(n)}(\sigma)$. 
Let $\gamma: I \rightarrow M_{q}^{4}(G)$ be a null curve with curvatures $k_{1}$ and $k_{2}$ and consider the set of vector fields along $\gamma$ whose components are polynomial functions

$$
\begin{gathered}
\mathfrak{X}_{\mathscr{P}}(\gamma)=\left\{V=f_{V} T+h_{V} W_{1}+g_{V} N+l_{V} W_{2}\right. \\
\left.\in \mathfrak{X}(\gamma): f_{V}, h_{V}, g_{V}, l_{V} \in \mathscr{P}\right\} .
\end{gathered}
$$

An element of $\mathfrak{X}_{\mathscr{P}}(\gamma)$ will be called a $\mathscr{P}$-local vector field along $\gamma$. The set of $\mathscr{P}$-local vector fields (locally preserving the causal character) will be denoted by

$$
\begin{aligned}
& \mathfrak{X}_{\mathscr{P}}^{*}(\gamma)=\left\{V=f_{V} T+h_{V} W_{1}+g_{V} N+l_{V} W_{2}\right. \\
& \left.\in \mathfrak{X}_{\mathscr{P}}(\gamma): g_{V}=-\varepsilon_{1} a D_{\sigma}^{-1}\left(h_{V}\right)\right\},
\end{aligned}
$$

and within it, the $\mathscr{P}$-local variation vector fields locally preserving pseudo arc-length parameter are described as

$$
\begin{aligned}
T_{\mathscr{P}, \gamma} \Lambda & =T_{\gamma}(\Lambda) \cap \mathfrak{X}_{\mathscr{P}}^{*}(\gamma)=\left\{V \in \mathfrak{X}_{\mathscr{P}}^{*}(\gamma): f_{V}\right. \\
& =-\frac{1}{2 a}\left[h_{V}^{\prime}+a k_{1} D_{\sigma}^{-1}\left(h_{V}\right)\right. \\
& \left.\left.-a D_{\sigma}^{-1}\left(k_{1} h_{V}-k_{2} l_{V}\right)\right]\right\} .
\end{aligned}
$$

In this context, from Proposition 5 and taking into account Remark 6, we can explicitly calculate the $\mathscr{P}$-local pseudo arc-length preserving variation vector fields by means of its constants of integration.

Proposition 7. Let $V$ be a vector field in $\mathfrak{X}_{\mathscr{P}}(\gamma)$, then $V \in$ $T_{\mathscr{P}, \gamma}(\Lambda)$ if and only if it is fulfilled that

$$
\begin{aligned}
g_{V} & =-\varepsilon_{1} a \partial_{\sigma}^{-1}\left(h_{V}\right)+c_{1}, \\
f_{V} & =-\frac{1}{2 a}\left[h_{V}^{\prime}+a k_{1} \partial_{\sigma}^{-1}\left(h_{V}\right)-a \partial_{\sigma}^{-1}\left(k_{1} h_{V}-k_{2} l_{V}\right)\right. \\
& \left.-\varepsilon_{1} c_{1} k_{1}\right]+c_{2},
\end{aligned}
$$

where $c_{1}, c_{2}$ are constants and $\partial_{\sigma}^{-1}$ is the antiderivative operator verifying that $\partial_{\sigma}^{-1} \circ \partial_{\sigma}=I$ when acting on $\mathscr{P}_{0}$.

Consequently, let us consider the set

$$
\begin{aligned}
\mathscr{Q} & =\left\{(h, l) \in \mathscr{P}_{0}^{2}: \exists(p, q) \in \mathscr{P}_{0}^{2} \text { such that }\left(p^{\prime}, q^{\prime}\right)\right. \\
& \left.=\left(h, k_{1} h-k_{2} l\right)\right\} .
\end{aligned}
$$

Given a pair of functions $(h, l) \in \mathbb{Q}$ and two constants $c_{1}$ and $c_{2}$, we will denote by $\mathscr{X}(h, l)$ the $\mathscr{P}$-local pseudo arc-length preserving variation vector field

$$
\begin{aligned}
& \mathscr{X}(h, l)=\left(-\frac{1}{2 a}\left[h^{\prime}+a k_{1} \partial_{\sigma}^{-1}(h)-a \partial_{\sigma}^{-1}\left(k_{1} h-k_{2} l\right)\right.\right. \\
& \left.\left.-\varepsilon_{1} c_{1} k_{1}\right]+c_{2}\right) T+h W_{1}+\left(-\varepsilon_{1} a \partial_{\sigma}^{-1}(h)+c_{1}\right) N \\
& +l W_{2} .
\end{aligned}
$$

Example 8. Consider the pair of functions $(h, l)=(0,0)$; then $\mathscr{X}(0,0)=\left(\left(\varepsilon_{1} c_{1} / 2 a\right) k_{1}+c_{2}\right) T+c_{1} N$. In particular, if we take $\left(c_{1}, c_{2}\right)=(0, b)$ and $\left(c_{1}, c_{2}\right)=\left(-2 \varepsilon_{1} a^{2} c, 0\right)$, where $b$ and $c$ are constants, one obtains the vector fields

$$
\begin{aligned}
& V_{0}=b T, \\
& V_{1}=-a c k_{1} T-2 \varepsilon_{1} a^{2} c N .
\end{aligned}
$$

The vector fields $V_{0}$ and $V_{1}$ will be the starting point of the commuting hierarchy of symmetries in Section 5.

Note that to introduce the concept of symmetry (and so a recursion operator) and furnish the phase space of null curve motions with a formal variational calculus in Section 5, an appropriate Lie bracket on the set of local vector fields should be defined. To this end, we first introduce a convenient derivation on both the differential algebra and the local vector fields along a null curve. Motivated by [32] and bearing in mind Lemma 4 , given $V \in \mathfrak{X}_{\mathscr{P}}^{*}(\gamma)$, we denote by $D_{V}$ : $\mathfrak{X}_{\mathscr{P}}(\gamma) \rightarrow \mathfrak{X}_{\mathscr{P}}(\gamma)$ the unique tensor derivation fulfilling:

$$
\begin{aligned}
& V\left(f^{\prime}\right)=V(f)^{\prime}+\frac{1}{2 a} \rho_{V} f^{\prime} \quad \forall f \in \mathscr{P} ; \\
& \left(\begin{array}{l}
V\left(k_{1}\right) \\
V\left(k_{2}\right)
\end{array}\right) \\
& =\left(\begin{array}{cc}
\frac{1}{a}\left(\frac{1}{a} D_{\sigma}^{3}+k_{1} D_{\sigma}+D_{\sigma} k_{1}\right) & -\frac{1}{a}\left(D_{\sigma} k_{2}+k_{2} D_{\sigma}\right) \\
\frac{1}{a}\left(D_{\sigma} k_{2}+k_{2} D_{\sigma}\right) & \frac{\varepsilon_{1} \varepsilon_{2}}{a}\left(\frac{1}{a} D_{\sigma}^{3}+k_{1} D_{\sigma}+D_{\sigma} k_{1}\right)
\end{array}\right) \\
& \cdot\left(\begin{array}{c}
\varphi_{V} \\
\psi_{V}
\end{array}\right)+\left(\begin{array}{c}
\frac{1}{a}\left(\frac{1}{2 a} \rho_{V}^{\prime \prime}+k_{1} \rho_{V}-2 G g_{V}^{\prime}\right) \\
\frac{1}{a} k_{2} \rho_{V}-\varepsilon_{2} G l_{V}
\end{array}\right) ; \\
& \left(\begin{array}{c}
D_{V} T \\
D_{V} W_{1} \\
D_{V} N \\
D_{V} W_{2}
\end{array}\right) \\
& =\left(\begin{array}{cccc}
-\alpha_{V} & \varphi_{V} & 0 & \psi_{V} \\
-\beta_{V} & 0 & \varepsilon_{1} \varphi_{V} & \frac{1}{a} \psi_{V}^{\prime} \\
0 & -\varepsilon_{1} \beta_{V} & \alpha_{V} & \frac{\varepsilon_{1}}{a} \delta_{V} \\
\frac{\varepsilon_{1} \varepsilon_{2}}{a} \delta_{V} & -\frac{\varepsilon_{1} \varepsilon_{2}}{a} \psi_{V}^{\prime} & \varepsilon_{2} \psi_{V} & 0
\end{array}\right) \cdot\left(\begin{array}{c}
T \\
W_{1} \\
N \\
W_{2}
\end{array}\right),
\end{aligned}
$$

where $\alpha_{V}, \beta_{V}$, and $\delta_{V}$ are given in (26). We now restrict our definition of Lie bracket only on the set $\mathfrak{X}_{\mathscr{P}}^{*}(\gamma)$, which will be enough for our purposes.

Proposition 9. Let $\gamma$ be a null curve in $\Lambda$ and consider $[\cdot, \cdot]_{\gamma}$ : $\mathfrak{X}_{\mathscr{P}}^{*}(\gamma) \times \mathfrak{X}_{\mathscr{P}}^{*}(\gamma) \rightarrow \mathfrak{X}_{\mathscr{P}}^{*}(\gamma)$ the map given by

$$
\left[V_{1}, V_{2}\right]_{\gamma}=D_{V_{1}} V_{2}-D_{V_{2}} V_{1} \text {. }
$$

Then the following holds:

(a) $[\cdot, \cdot]_{\gamma}$ is well defined; that is, if $V_{1}, V_{2} \in \mathfrak{X}_{\mathscr{P}}^{*}(\gamma)$ then $\left[V_{1}, V_{2}\right]_{\gamma} \in \mathfrak{X}_{\mathscr{P}}^{*}(\gamma)$. 
(b) $\left[V_{1}, V_{2}\right]_{\gamma}(f)=V_{1} V_{2}(f)-V_{2} V_{1}(f)$ for all $f \in \mathscr{P}$.

(c) $[\cdot, \cdot]_{\gamma}$ is skew-symmetric.

(d) For $V_{1}, V_{2} \in \mathfrak{X}_{\mathscr{P}}^{*}(\gamma)$ and $U \in \mathfrak{X}_{\mathscr{P}}(\gamma)$ we have

$$
\begin{array}{r}
D_{\left[V_{1}, V_{2}\right]} U-D_{V_{1}} D_{V_{2}} U+D_{V_{2}} D_{V_{1}} U \\
=G\left(\left\langle U, V_{1}\right\rangle V_{2}-\left\langle U, V_{2}\right\rangle V_{1}\right) .
\end{array}
$$

(e) $[\cdot, \cdot]_{\gamma}$ satisfies the Jacobi identity.

(f) $[\cdot, \cdot]_{\gamma}$ is closed for elements in $T_{\mathscr{P}, \gamma}(\Lambda)$, that is, if $V, U \in$ $T_{\mathscr{P}, \gamma}(\Lambda)$, then $[V, U]_{\gamma} \in T_{\mathscr{P}, \gamma}(\Lambda)$.

Proof. Given two vector fields $V_{1}=f_{1} T+h_{1} W_{1}+g_{1} N+l_{1} W_{2}$ and $V_{2}=f_{2} T+h_{2} W_{1}+g_{2} N+l_{2} W_{2}$ in $\mathfrak{X}_{\mathscr{P}}^{*}(\gamma)$, set $V_{12}=$ $\left[V_{1}, V_{2}\right]_{\gamma}$. The components $g_{12}$ and $h_{12}$ of $V_{12}$ are given by

$$
\begin{aligned}
g_{12}= & V_{1}\left(g_{2}\right)-V_{2}\left(g_{1}\right)-\frac{1}{a}\left(\varphi_{1} g_{2}^{\prime}-\varphi_{2} g_{1}^{\prime}\right) \\
& +\left(\alpha_{1} g_{2}-\alpha_{2} g_{1}\right)+\varepsilon_{2}\left(l_{2} \psi_{1}-l_{1} \psi_{2}\right), \\
h_{12}= & \frac{\varepsilon_{1}}{a}\left(V_{2}\left(g_{1}^{\prime}\right)-V_{1}\left(g_{2}^{\prime}\right)\right)+\left(\varphi_{1} f_{2}-\varphi_{2} f_{1}\right) \\
& +\varepsilon_{1}\left(\beta_{2} g_{1}-\beta_{1} g_{2}\right)+\frac{\varepsilon_{1} \varepsilon_{2}}{a}\left(l_{1} \psi_{2}^{\prime}-l_{2} \psi_{1}^{\prime}\right) .
\end{aligned}
$$

Since $V_{1}, V_{2} \in \mathfrak{X}_{\mathscr{P}}^{*}(\gamma)$, they verify formulas (35) and (42) which, together with definitions of $\alpha_{i}$ and $\beta_{i}$, lead to the relation $g_{12}^{\prime}=-a \varepsilon_{1} h_{12}$. The latter is the condition equivalent to $\left[V_{1}, V_{2}\right]_{\gamma} \in \mathfrak{X}_{\mathscr{P}}^{*}(\gamma)$, thus proving (a). To prove (b), it is sufficient to check the same equality solely for generators $k_{1}$ and $k_{2}$ of algebra $\mathscr{P}$. We will calculate the expressions of $\varphi_{12}, \psi_{12}$, and $\rho_{12}$ (corresponding functions to the Lie bracket $\left[V_{1}, V_{2}\right]_{\gamma}$ ), by means of $\varphi_{i}, \psi_{i}$, and $\rho_{i}$ (corresponding functions to vector fields $\left.V_{i}\right)$. If $V=f_{V} T+h_{V} W_{1}+g_{V} N+l_{V} W_{2} \in \mathfrak{X}_{\mathscr{P}}(\gamma)$ is any vector field, we have

$$
\begin{aligned}
& \varphi_{V}=a f_{V}+h_{V}^{\prime}-\varepsilon_{1} k_{1} g_{V}, \\
& \psi_{V}=l_{V}^{\prime}+\varepsilon_{2} k_{2} g_{V}, \\
& \rho_{V}=-a f_{V}^{\prime}+2 a k_{1} h_{V}-a k_{2} l_{V}-\varphi_{V}^{\prime}+\varepsilon_{1} k_{1} g_{V}^{\prime}, \\
& h_{V}=-\frac{\varepsilon_{1}}{a} g_{V}^{\prime}
\end{aligned}
$$

$$
\text { if } V \in \mathfrak{X}_{\mathscr{P}}^{*}(\gamma) \text {. }
$$

Bearing in mind relations (61) and expressions of $V\left(k_{1}\right)$ and $V\left(k_{2}\right)$ obtained in Lemma 4, we deduce

$$
\begin{aligned}
\varphi_{D_{V_{1}} V_{2}} & =a f_{D_{V_{1}} V_{2}}+h_{D_{V_{1}} V_{2}}^{\prime}-\varepsilon_{1} k_{1} g_{D_{V_{1}} V_{2}} \\
= & V_{1}\left(\varphi_{2}\right)-\frac{1}{2 a} \rho_{1} \varphi_{2}-\frac{1}{a} \varphi_{1} \rho_{2}-\frac{1}{a} \varphi_{1} \varphi_{2}^{\prime}-\frac{\varepsilon_{1} \varepsilon_{2}}{a} \psi_{1}^{\prime} \psi_{2} \\
& -\frac{\varepsilon_{1}}{a} G g_{1}^{\prime} g_{2} ;
\end{aligned}
$$

$$
\begin{aligned}
\psi_{D_{V_{1}} V_{2}} & =l_{D_{V_{1}} V_{2}}^{\prime}+\varepsilon_{2} k_{2} g_{D_{V_{1}} V_{2}} \\
= & V_{1}\left(\psi_{2}\right)-\frac{1}{2 a} \rho_{1} \psi_{2}-\frac{1}{a} \rho_{2} \psi_{1}+\frac{1}{a}\left(\psi_{1}^{\prime} \varphi_{2}-\psi_{1} \varphi_{2}^{\prime}\right) \\
& +G l_{1} g_{2} ; \\
\rho_{D_{V_{1}} V_{2}} & \\
= & -a f_{D_{V_{1}} V_{2}}^{\prime}+2 a k_{1} h_{D_{V_{1}} V_{2}}-a k_{2} l_{D_{V_{1}} V_{2}}-\varphi_{D_{V_{1}} V_{2}}^{\prime} \\
& +\varepsilon_{1} k_{1} g_{D_{V_{1}} V_{2}}^{\prime} \\
= & V_{1}\left(\rho_{2}\right)-\frac{1}{a} \rho_{1} \rho_{2}+\frac{1}{a}\left(\rho_{1}^{\prime} \varphi_{2}+\varphi_{1} \rho_{2}^{\prime}\right) \\
& +\frac{1}{a}\left(\varphi_{1}^{\prime \prime} \varphi_{2}+\varphi_{2}^{\prime \prime} \varphi_{1}\right)+2 k_{1} \varphi_{1} \varphi_{2} \\
& -k_{2}\left(\varphi_{1} \psi_{2}+\varphi_{2} \psi_{1}\right)+\frac{\varepsilon_{1} \varepsilon_{2}}{a} \psi_{1}^{\prime} \psi_{2}^{\prime} \\
& +G\left(\frac{\varepsilon_{1}}{a}\left(g_{1} g_{2}^{\prime \prime}+g_{2} g_{1}^{\prime \prime}\right)+2 \varepsilon_{1} k_{1} g_{1} g_{2}-a \varepsilon_{2} l_{1} l_{2}\right) .
\end{aligned}
$$

Because of symmetry of formulas (62), deleting terms with repeated factors and rearranging the other, we obtain

$$
\begin{aligned}
\varphi_{12}= & V_{1}\left(\varphi_{2}\right)-V_{2}\left(\varphi_{1}\right)+\frac{1}{2 a}\left(\rho_{1} \varphi_{2}-\rho_{2} \varphi_{1}\right) \\
& +\frac{1}{a}\left(\varphi_{1}^{\prime} \varphi_{2}-\varphi_{1} \varphi_{2}^{\prime}\right)+\frac{\varepsilon_{1} \varepsilon_{2}}{a}\left(\psi_{1} \psi_{2}^{\prime}-\psi_{1}^{\prime} \psi_{2}\right) \\
& +\frac{\varepsilon_{1}}{a} G\left(g_{2}^{\prime} g_{1}-g_{2} g_{1}^{\prime}\right), \\
\psi_{12}= & V_{1}\left(\psi_{2}\right)-V_{2}\left(\psi_{1}\right)+\frac{1}{2 a}\left(\rho_{1} \psi_{2}-\rho_{2} \psi_{1}\right) \\
& +\frac{1}{a}\left(\psi_{1}^{\prime} \varphi_{2}-\psi_{1} \varphi_{2}^{\prime}+\psi_{2} \varphi_{1}^{\prime}-\psi_{2}^{\prime} \varphi_{1}\right) \\
& +G\left(l_{1} g_{2}-l_{2} g_{1}\right), \\
\rho_{12}= & V_{1}\left(\rho_{2}\right)-V_{2}\left(\rho_{1}\right) .
\end{aligned}
$$

From Lemma 4 we obtain

$$
\begin{aligned}
{\left[V_{1}, V_{2}\right]_{\gamma}\left(k_{1}\right)=} & \frac{1}{a^{2}} \varphi_{12}^{\prime \prime \prime}+\frac{1}{a}\left(k_{1}^{\prime} \varphi_{12}+2 k_{1} \varphi_{12}^{\prime}\right) \\
& -\frac{1}{a}\left(k_{2}^{\prime} \psi_{12}+2 k_{2} \psi_{12}^{\prime}\right) \\
& +\frac{1}{a}\left(\frac{1}{2 a} \rho_{12}^{\prime \prime}+k_{1} \rho_{12}-2 G g_{12}^{\prime}\right) .
\end{aligned}
$$

Expanding each earlier term by using $V(f)^{\prime \prime \prime}=V\left(f^{\prime \prime \prime}\right)-$ $(3 / 2 a) \rho_{V} f^{\prime \prime \prime}-(3 / 2 a) \rho_{V}^{\prime} f^{\prime \prime}-(1 / 2 a) \rho_{V}^{\prime \prime} f^{\prime}$ and other properties it follows that

$$
\begin{gathered}
\frac{1}{a^{2}} \varphi_{12}^{\prime \prime \prime}=V_{1}\left(\frac{1}{a^{2}} \varphi_{2}^{\prime \prime \prime}\right)-V_{2}\left(\frac{1}{a^{2}} \varphi_{1}^{\prime \prime \prime}\right)+\frac{1}{a^{3}}\left(\rho_{2} \varphi_{1}^{\prime \prime \prime}\right. \\
\left.-\rho_{1} \varphi_{2}^{\prime \prime \prime}\right)+\frac{1}{a^{3}}\left(\rho_{1}^{\prime \prime} \varphi_{2}^{\prime}-\rho_{2}^{\prime \prime} \varphi_{1}^{\prime}\right)+\frac{1}{2 a^{3}}\left(\rho_{1}^{\prime \prime \prime} \varphi_{2}\right.
\end{gathered}
$$




$$
\begin{aligned}
& \left.-\rho_{2}^{\prime \prime \prime} \varphi_{1}\right)+\frac{1}{a^{3}}\left(\varphi_{1}^{(4)} \varphi_{2}-\varphi_{1} \varphi_{2}^{(4)}\right. \\
& \left.+2\left(\varphi_{1}^{\prime \prime \prime} \varphi_{2}^{\prime}-\varphi_{2}^{\prime \prime \prime} \varphi_{1}^{\prime}\right)\right)+\frac{\varepsilon_{1} \varepsilon_{2}}{a^{3}}\left(\psi_{1} \psi_{2}^{(4)}-\psi_{2} \psi_{1}^{(4)}\right. \\
& \left.+2\left(\psi_{1}^{\prime} \psi_{2}^{\prime \prime \prime}-\psi_{2}^{\prime} \psi_{1}^{\prime \prime \prime}\right)\right)+\frac{\varepsilon_{1}}{a^{3}} G\left(g_{2}^{\prime} g_{1}-g_{2} g_{1}^{\prime}\right)^{\prime \prime \prime} ; \\
& \frac{1}{a}\left(k_{1}^{\prime} \varphi_{12}+2 k_{1} \varphi_{12}^{\prime}\right)=V_{1}\left(\frac{1}{a}\left(k_{1}^{\prime} \varphi_{2}+2 k_{1} \varphi_{2}^{\prime}\right)\right) \\
& -V_{2}\left(\frac{1}{a}\left(k_{1}^{\prime} \varphi_{1}+2 k_{1} \varphi_{1}^{\prime}\right)\right)+\frac{1}{a}\left(V_{2}\left(2 k_{1}\right) \varphi_{1}^{\prime}\right. \\
& \left.+V_{2}\left(k_{1}^{\prime}\right) \varphi_{1}-V_{1}\left(2 k_{1}\right) \varphi_{2}^{\prime}-V_{1}\left(k_{1}^{\prime}\right) \varphi_{2}\right) \\
& +\frac{k_{1}}{a^{2}}\left(\rho_{1}^{\prime} \varphi_{2}-\rho_{2}^{\prime} \varphi_{1}\right)+\frac{2 k_{1}}{a^{2}}\left(\varphi_{2} \varphi_{1}^{\prime \prime}-\varphi_{1} \varphi_{2}^{\prime \prime}\right) \\
& +\frac{2 \varepsilon_{1} \varepsilon_{2} k_{1}}{a^{2}}\left(\psi_{1} \psi_{2}^{\prime \prime}-\psi_{2} \psi_{1}^{\prime \prime}\right)+\frac{k_{1}^{\prime}}{2 a^{2}}\left(\rho_{1} \varphi_{2}-\rho_{2} \varphi_{1}\right) \\
& +\frac{k_{1}^{\prime}}{a^{2}}\left(\varphi_{2} \varphi_{1}^{\prime}-\varphi_{1} \varphi_{2}^{\prime}\right)+\frac{\varepsilon_{1} \varepsilon_{2} k_{1}^{\prime}}{a^{2}}\left(\psi_{1} \psi_{2}^{\prime}-\psi_{2} \psi_{1}^{\prime}\right) \\
& +\frac{2 \varepsilon_{1} k_{1}}{a^{2}} G\left(g_{2}^{\prime} g_{1}-g_{2} g_{1}^{\prime}\right)^{\prime}+\frac{\varepsilon_{1} k_{1}^{\prime}}{a^{2}} G\left(g_{2}^{\prime} g_{1}-g_{2} g_{1}^{\prime}\right) ; \\
& -\frac{1}{a}\left(k_{2}^{\prime} \psi_{12}+2 k_{2} \psi_{12}^{\prime}\right)=V_{1}\left(-\frac{1}{a}\left(k_{2}^{\prime} \psi_{2}+2 k_{2} \psi_{2}^{\prime}\right)\right) \\
& -V_{2}\left(-\frac{1}{a}\left(k_{2}^{\prime} \psi_{1}+2 k_{2} \psi_{1}^{\prime}\right)\right)-\frac{1}{a}\left(V_{2}\left(2 k_{2}\right) \psi_{1}^{\prime}\right. \\
& \left.+V_{2}\left(k_{2}^{\prime}\right) \psi_{1}-V_{1}\left(2 k_{2}\right) \psi_{2}^{\prime}-V_{1}\left(k_{2}^{\prime}\right) \psi_{2}\right) \\
& -\frac{k_{2}}{a^{2}}\left(\rho_{1}^{\prime} \psi_{2}-\rho_{2}^{\prime} \psi_{1}\right)-\frac{k_{2}^{\prime}}{2 a^{2}}\left(\rho_{1} \psi_{2}-\rho_{2} \psi_{1}\right) \\
& -\frac{2 k_{2}}{a^{2}}\left(\varphi_{2} \psi_{1}^{\prime \prime}-\psi_{1} \varphi_{2}^{\prime \prime}+\psi_{2} \varphi_{1}^{\prime \prime}-\psi_{2}^{\prime \prime} \varphi_{1}\right) \\
& -\frac{k_{2}^{\prime}}{a^{2}}\left(\psi_{1}^{\prime} \varphi_{2}-\psi_{1} \varphi_{2}^{\prime}+\psi_{2} \varphi_{1}^{\prime}-\psi_{2}^{\prime} \varphi_{1}\right)-\frac{k_{2}^{\prime}}{a} G\left(l_{1} g_{2}\right. \\
& \left.-l_{2} g_{1}\right)-\frac{2 k_{2}}{a} G\left(l_{1} g_{2}-l_{2} g_{1}\right)^{\prime} \text {. }
\end{aligned}
$$

Based on the expressions $V(f)^{\prime \prime}=V\left(f^{\prime \prime}\right)-(1 / a) \rho_{V} f^{\prime \prime}-$ $(1 / 2 a) \rho_{V}^{\prime} f^{\prime}$ and $V(f)^{\prime}=V\left(f^{\prime}\right)-(1 / 2 a) \rho_{V} f^{\prime}$ it is also easy to establish that

$$
\begin{aligned}
\frac{1}{2 a^{2}} \rho_{12}^{\prime \prime}= & V_{1}\left(\frac{1}{2 a^{2}} \rho_{2}^{\prime \prime}\right)-V_{2}\left(\frac{1}{2 a^{2}} \rho_{1}^{\prime \prime}\right) \\
& +\frac{1}{2 a^{3}}\left(\rho_{2} \rho_{1}^{\prime \prime}-\rho_{1} \rho_{2}^{\prime \prime}\right) \\
\frac{k_{1}}{a} \rho_{12}= & V_{1}\left(\frac{k_{1}}{a} \rho_{2}\right)-V_{2}\left(\frac{k_{1}}{a} \rho_{1}\right)+V_{2}\left(\frac{k_{1}}{a}\right) \rho_{1} \\
& -V_{1}\left(\frac{k_{1}}{a}\right) \rho_{2},
\end{aligned}
$$

$$
\begin{aligned}
-\frac{2 G}{a} g_{12}^{\prime}= & V_{1}\left(-\frac{2 G}{a} g_{2}^{\prime}\right)-V_{2}\left(-\frac{2 G}{a} g_{1}^{\prime}\right) \\
& +\frac{G}{a^{2}}\left(\rho_{1} g_{2}^{\prime}-\rho_{2} g_{1}^{\prime}\right) \\
& +\frac{2 G}{a^{2}}\left(\varphi_{1}^{\prime} g_{2}^{\prime}-\varphi_{2}^{\prime} g_{1}^{\prime}+\varphi_{1} g_{2}^{\prime \prime}-\varphi_{2} g_{1}^{\prime \prime}\right) \\
& -\frac{2 G}{a}\left(\alpha_{1}^{\prime} g_{2}+\alpha_{1} g_{2}^{\prime}-\alpha_{2}^{\prime} g_{1}-\alpha_{2} g_{1}^{\prime}\right) \\
& -\frac{2 \varepsilon_{2} G}{a}\left(\psi_{1}^{\prime} l_{2}+\psi_{1} l_{2}^{\prime}-l_{1}^{\prime} \psi_{2}-l_{1} \psi_{2}^{\prime}\right) .
\end{aligned}
$$

When adding up (64), (65), and (66) and making a long but easy computation we obtain

$$
\left[V_{1}, V_{2}\right]_{\gamma}\left(k_{1}\right)=V_{1} V_{2}\left(k_{1}\right)-V_{2} V_{1}\left(k_{1}\right)
$$

Using again Lemma 4 we have

$$
\begin{aligned}
{\left[V_{1}, V_{2}\right]_{\gamma}\left(k_{2}\right)=} & \frac{\varepsilon_{1} \varepsilon_{2}}{a^{2}} \psi_{12}^{\prime \prime \prime}+\frac{\varepsilon_{1} \varepsilon_{2}}{a}\left(k_{1}^{\prime} \psi_{12}+2 k_{1} \psi_{12}^{\prime}\right) \\
& +\frac{1}{a}\left(k_{2}^{\prime} \varphi_{12}+2 k_{2} \varphi_{12}^{\prime}\right) \\
& +\frac{1}{a}\left(k_{2} \rho_{12}-a \varepsilon_{2} G l_{12}\right)
\end{aligned}
$$

In the same way as (63), we can compute the terms of (68),

$$
\begin{aligned}
& \frac{\varepsilon_{1} \varepsilon_{2}}{a^{2}} \psi_{12}^{\prime \prime \prime}=V_{1}\left(\frac{\varepsilon_{1} \varepsilon_{2}}{a^{2}} \psi_{2}^{\prime \prime \prime}\right)-V_{2}\left(\frac{\varepsilon_{1} \varepsilon_{2}}{a^{2}} \psi_{1}^{\prime \prime \prime}\right) \\
& +\frac{\varepsilon_{1} \varepsilon_{2}}{a^{3}}\left(\rho_{1}^{\prime \prime} \psi_{2}^{\prime}-\rho_{2}^{\prime \prime} \psi_{1}^{\prime}\right)+\frac{\varepsilon_{1} \varepsilon_{2}}{2 a^{3}}\left(\rho_{1}^{\prime \prime \prime} \psi_{2}-\rho_{2}^{\prime \prime \prime} \psi_{1}\right) \\
& +\frac{\varepsilon_{1} \varepsilon_{2}}{a^{3}}\left[\psi_{1}^{(4)} \varphi_{2}-\psi_{2}^{(4)} \varphi_{1}+2\left(\psi_{1}^{\prime \prime \prime} \varphi_{2}^{\prime}-\psi_{2}^{\prime \prime \prime} \varphi_{1}^{\prime}\right)\right. \\
& \left.+2\left(\psi_{2}^{\prime} \varphi_{1}^{\prime \prime \prime}-\psi_{1}^{\prime} \varphi_{2}^{\prime \prime \prime}\right)+\left(\psi_{2} \varphi_{1}^{(4)}-\psi_{1} \varphi_{2}^{(4)}\right)\right]+\frac{\varepsilon_{1} \varepsilon_{2}}{a^{2}} \\
& +G\left(l_{1} g_{2}-l_{2} g_{1}\right)^{\prime \prime \prime} ; \\
& \frac{\varepsilon_{1} \varepsilon_{2}}{a}\left(k_{1}^{\prime} \psi_{12}+2 k_{1} \psi_{12}^{\prime}\right)=V_{1}\left(\frac{\varepsilon_{1} \varepsilon_{2}}{a}\left(k_{1}^{\prime} \psi_{2}+2 k_{1} \psi_{2}^{\prime}\right)\right) \\
& \quad-V_{2}\left(\frac{\varepsilon_{1} \varepsilon_{2}}{a}\left(k_{1}^{\prime} \psi_{1}+2 k_{1} \psi_{1}^{\prime}\right)\right)+\frac{\varepsilon_{1} \varepsilon_{2}}{a}\left(V_{2}\left(2 k_{1}\right) \psi_{1}^{\prime}\right. \\
& \left.+V_{2}\left(k_{1}^{\prime}\right) \psi_{1}-V_{1}\left(2 k_{1}\right) \psi_{2}^{\prime}-V_{1}\left(k_{1}^{\prime}\right) \psi_{2}\right) \\
& +\frac{\varepsilon_{1} \varepsilon_{2} k_{1}}{a^{2}}\left(\rho_{1}^{\prime} \psi_{2}-\rho_{2}^{\prime} \psi_{1}\right)+\frac{\varepsilon_{1} \varepsilon_{2} k_{1}^{\prime}}{2 a^{2}}\left(\rho_{1} \psi_{2}-\rho_{2} \psi_{1}\right) \\
& a^{2}\left(\varphi_{2} \psi_{1}^{\prime \prime}-\varphi_{1} \psi_{2}^{\prime \prime}+\psi_{2} \varphi_{1}^{\prime \prime}-\psi_{1} \varphi_{2}^{\prime \prime}\right)
\end{aligned}
$$




$$
\begin{aligned}
& +\frac{\varepsilon_{1} \varepsilon_{2} k_{1}^{\prime}}{a^{2}}\left(\varphi_{2} \psi_{1}^{\prime}-\psi_{1} \varphi_{2}^{\prime}+\psi_{2} \varphi_{1}^{\prime}-\psi_{2}^{\prime} \varphi_{1}\right) \\
& +\frac{\varepsilon_{1} \varepsilon_{2} G}{a}\left[2 k_{1}\left(l_{1} g_{2}-l_{2} g_{1}\right)^{\prime}+k_{1}^{\prime}\left(l_{1} g_{2}-l_{2} g_{1}\right)\right] \\
& \frac{1}{a}\left(k_{2}^{\prime} \varphi_{12}+2 k_{2} \varphi_{12}^{\prime}\right)=V_{1}\left(\frac{1}{a}\left(k_{2}^{\prime} \varphi_{2}+2 k_{2} \varphi_{2}^{\prime}\right)\right) \\
& -V_{2}\left(\frac{1}{a}\left(k_{2}^{\prime} \varphi_{1}+2 k_{2} \varphi_{1}^{\prime}\right)\right)+\frac{1}{a}\left(V_{2}\left(2 k_{2}\right) \varphi_{1}^{\prime}\right. \\
& \left.+V_{2}\left(k_{2}^{\prime}\right) \varphi_{1}-V_{1}\left(2 k_{2}\right) \varphi_{2}^{\prime}-V_{1}\left(k_{2}^{\prime}\right) \varphi_{2}\right) \\
& +\frac{k_{2}}{a^{2}}\left(\rho_{1}^{\prime} \varphi_{2}-\rho_{2}^{\prime} \varphi_{1}\right)+\frac{k_{2}^{\prime}}{2 a^{2}}\left(\rho_{1} \varphi_{2}-\rho_{2} \varphi_{1}\right) \\
& +\frac{2 k_{2}}{a^{2}}\left(\varphi_{2} \varphi_{1}^{\prime \prime}-\varphi_{1} \varphi_{2}^{\prime \prime}\right)+\frac{2 \varepsilon_{1} \varepsilon_{2} k_{2}}{a^{2}}\left(\psi_{1} \psi_{2}^{\prime \prime}-\psi_{1}^{\prime \prime} \psi_{2}\right) \\
& +\frac{k_{2}^{\prime}}{a^{2}}\left(\varphi_{1}^{\prime} \varphi_{2}-\varphi_{1} \varphi_{2}^{\prime}\right)+\frac{\varepsilon_{1} \varepsilon_{2} k_{2}^{\prime}}{a^{2}}\left(\psi_{1} \psi_{2}^{\prime}-\psi_{1}^{\prime} \psi_{2}\right) \\
& +\frac{\varepsilon_{1} G}{a^{2}}\left[2 k_{2}\left(g_{2}^{\prime} g_{1}-g_{2} g_{1}^{\prime}\right)^{\prime}+k_{2}^{\prime}\left(g_{2}^{\prime} g_{1}-g_{2} g_{1}^{\prime}\right)\right] ; \\
& \frac{k_{2}}{a} \rho_{12}=V_{1}\left(\frac{k_{2}}{a} \rho_{2}\right)-V_{2}\left(\frac{k_{2}}{a} \rho_{1}\right)+V_{2}\left(\frac{k_{2}}{a}\right) \rho_{1} \\
& -V_{1}\left(\frac{k_{1}}{a}\right) \rho_{2} \\
& -\varepsilon_{2} G l_{12}=V_{1}\left(-\varepsilon_{2} G l_{2}\right)-V_{2}\left(-\varepsilon_{2} G l_{1}\right)-\varepsilon_{2} G\left(f_{2} \psi_{1}\right. \\
& \left.-f_{1} \psi_{2}\right)+\frac{\varepsilon_{1} \varepsilon_{2}}{a^{2}}\left(g_{2}^{\prime} \psi_{1}^{\prime}-g_{1}^{\prime} \psi_{2}^{\prime}\right)-\frac{\varepsilon_{1} \varepsilon_{2} G}{a}\left(g_{2} \delta_{1}\right. \\
& \left.-g_{1} \delta_{2}\right)
\end{aligned}
$$

After some work, it also follows from (69) that

$$
\left[V_{1}, V_{2}\right]_{\gamma}\left(k_{2}\right)=V_{1} V_{2}\left(k_{2}\right)-V_{2} V_{1}\left(k_{2}\right) \text {. }
$$

Paragraph (c) is a direct consequence of the definition and (f) is also trivial taking into account the expression for $\rho_{12}$ given in (63). Paragraph (d) follows from a straightforward computation. Finally, to prove (e), let us denote $R\left(V_{1}, V_{2}\right) U=$ $D_{\left[V_{1}, V_{2}\right]} U-D_{V_{1}} D_{V_{2}} U+D_{V_{2}} D_{V_{1}} U$. By using (d) we obtain

$$
\begin{aligned}
& {\left[\left[V_{1}, V_{2}\right], V_{3}\right]+\left[\left[V_{2}, V_{3}\right], V_{1}\right]+\left[\left[V_{3}, V_{1}\right], V_{2}\right]} \\
& \quad=R\left(V_{1}, V_{2}\right) V_{3}+R\left(V_{2}, V_{3}\right) V_{1}+R\left(V_{3}, V_{1}\right) V_{2}=0 .
\end{aligned}
$$

In particular, Proposition 9 entails that the set of $\mathscr{P}$-local pseudo arc-length preserving variation vector fields $T_{\mathscr{P}, \gamma}(\Lambda)$ is a Lie subalgebra of the Lie algebra of the $\mathscr{P}$-local vector fields $\left(\mathfrak{X}_{\mathscr{P}}^{*}(\gamma),[\cdot, \cdot]_{\gamma}\right)$.

Before turning to study the geometric hierarchies of null curve flows in Section 5, we point out that (36) and (56) are particularly noteworthy when vector fields locally preserve the pseudo arc-length parameter and curvature $G$ vanishes. Equations (36) and (56) may be rewritten as

$$
\begin{aligned}
\left(\varphi_{V}, \psi_{V}\right) & =A\left(h_{V}, l_{V}\right) \\
& =\left(A_{1}\left(h_{V}, l_{V}\right), A_{2}\left(h_{V}, l_{V}\right)\right), \\
\left(V\left(k_{1}\right), V\left(k_{2}\right)\right) & =B\left(\varphi_{V}, \psi_{V}\right) \\
& =\left(B_{1}\left(\varphi_{V}, \psi_{V}\right), B_{2}\left(\varphi_{V}, \psi_{V}\right)\right),
\end{aligned}
$$

where

$$
\begin{aligned}
& A=\left(\begin{array}{l}
A_{1} \\
A_{2}
\end{array}\right)=\left(\begin{array}{cc}
\frac{a}{2} \omega\left(k_{1}\right) & -\frac{a}{2} D_{\sigma}^{-1} k_{2} \\
-\varepsilon_{1} \varepsilon_{2} a k_{2} D_{\sigma}^{-1} & D_{\sigma}
\end{array}\right) ; \\
& B=\left(\begin{array}{l}
B_{1} \\
B_{2}
\end{array}\right)=\frac{1}{a}\left(\begin{array}{cc}
\theta\left(k_{1}\right) & -S\left(k_{2}\right) \\
S\left(k_{2}\right) & \varepsilon_{1} \varepsilon_{2} \theta\left(k_{1}\right)
\end{array}\right),
\end{aligned}
$$

with $\omega\left(k_{1}\right)=(1 / a) D_{\sigma}+k_{1} D_{\sigma}^{-1}+D_{\sigma}^{-1} k_{1}, \theta\left(k_{1}\right)=(1 / a) D_{\sigma}^{3}+$ $k_{1} D_{\sigma}+D_{\sigma} k_{1}$, and $S\left(k_{2}\right)=D_{\sigma} k_{2}+k_{2} D_{\sigma}$. It should be remarked that $A$ and $B$ come very close to being the symplectic and cosymplectic operators, respectively, for (up to scaling) the Hirota-Satsuma system (see [12, 13]). Equation (72) can also be regarded as

$$
\begin{aligned}
& \left(\varphi_{V},-\psi_{V}\right)=J\left(2 h_{V},-\varepsilon_{1} \varepsilon_{2} l_{V}\right) \\
& =\left(J_{1}\left(2 h_{V},-\varepsilon_{1} \varepsilon_{2} l_{V}\right), J_{2}\left(2 h_{V},-\varepsilon_{1} \varepsilon_{2} l_{V}\right)\right), \\
& \left(V\left(k_{1}\right), V\left(k_{2}\right)\right)=\Theta\left(\varphi_{V},-\psi_{V}\right) \\
& \quad=\left(\Theta_{1}\left(\varphi_{V},-\psi_{V}\right), \Theta_{2}\left(\varphi_{V},-\psi_{V}\right)\right),
\end{aligned}
$$

where

$$
\begin{aligned}
& J=\left(\begin{array}{l}
J_{1} \\
J_{2}
\end{array}\right)=-\frac{\varepsilon_{1} \varepsilon_{2}}{2}\left(\begin{array}{cc}
-\frac{\varepsilon_{1} \varepsilon_{2} a}{2} \omega\left(k_{1}\right) & -a D_{\sigma}^{-1} k_{2} \\
-a k_{2} D_{\sigma}^{-1} & -2 D_{\sigma}
\end{array}\right) ; \\
& \Theta=\left(\begin{array}{l}
\Theta_{1} \\
\Theta_{2}
\end{array}\right)=\frac{1}{a}\left(\begin{array}{cc}
\theta\left(k_{1}\right) & S\left(k_{2}\right) \\
S\left(k_{2}\right) & -\varepsilon_{1} \varepsilon_{2} \theta\left(k_{1}\right)
\end{array}\right) .
\end{aligned}
$$

It is now therefore evident that $J$ and $\Theta$ are the symplectic and cosymplectic operators, respectively, for a rescaling of the HS-cKdV system. They have been obtained in a natural way using projections onto the screen bundle of both, the variation vector field $V$ and its covariant derivative $\nabla_{T} V$. This allows us automatically to determine the recursion operator $R=\Theta \circ J$, and the crucial relation

$$
\left(V\left(k_{1}\right), V\left(k_{2}\right)\right)=R\left(2 h_{V},-\varepsilon_{1} \varepsilon_{2} l_{V}\right) .
$$

Somewhat analogous relationships were obtained between curve evolution in 3-dimensional Riemannian manifolds in [19] (or more generally in $n$-dimensional Riemannian manifold with constant curvature in [20]) and the mKdV system. The above connection together with the availability of the Lie bracket provided by Proposition 9 will be employed to study the integrability of null curve evolution in the next section. 


\section{Geometric Hierarchies of Null Curve Flows}

The background given in [1] for the 3-dimensional case used to construct a commuting hierarchy for null curve evolutions can also be well adapted to the 4-dimensional case. Consider $\Lambda$ the space of pseudo arc-length parametrized null curves in the pseudo-Euclidean space $\mathbb{R}_{q}^{4}$. A map $\mathbf{f}: \Lambda \rightarrow \mathscr{C}^{\infty}(I, \mathbb{R})$ is referred to a scalar field on $\Lambda$ and $\mathbf{f}(\gamma)$ will be also denoted by $\mathbf{f}_{\gamma}$. Let $\mathscr{A}$ be the algebra of $\mathscr{P}$-valued scalar fields on $\Lambda$, that is, if $\mathbf{f} \in \mathscr{A}$, then $\mathbf{f}_{\gamma} \in \mathscr{P}$ for all $\gamma \in \Lambda$. In this sense, we will also understand the curvatures scalar fields $\mathbf{k}_{1}, \mathbf{k}_{2}: \Lambda \rightarrow$ $\mathscr{C}^{\infty}(I, \mathbb{R})$ with its obvious meaning.

Similarly, a map V $: \Lambda \rightarrow \bigcup_{\gamma \in \Lambda} T_{\gamma} \Lambda$ is referred to as a vector field on $\Lambda$, and $\mathbf{V}(\gamma)$ will be also denoted by $\mathbf{V}_{\gamma}$. We will denote the set of tangent vector fields on $\Lambda$ as $\mathfrak{X}(\Lambda)$, and within we consider the subset $\mathfrak{X}_{\mathscr{A}}(\Lambda)$ of vector fields $\mathbf{V}$ such that $\mathbf{V}_{\gamma} \in \mathfrak{X}_{\mathscr{P}}(\gamma)$; namely, if we denote $\mathbf{V}=\mathbf{f} \mathbf{T}+\mathbf{h W} \mathbf{W}_{\mathbf{1}}+\mathbf{g N}+$ $\mathbf{I W}_{2}$, then

$$
\begin{aligned}
& \mathfrak{X}_{\mathscr{A}}(\Lambda)=\left\{\mathbf{v} \in \mathfrak{X}(\Lambda): \mathbf{f}, \mathbf{h}, \mathbf{g}, \mathbf{l} \in \mathscr{A} ; \mathbf{h}=-\frac{\varepsilon_{1}}{a} \mathbf{g}^{\prime} ; \mathbf{f}\right. \\
& \left.\quad=\frac{1}{2 a}\left[\frac{\varepsilon_{1}}{a} \mathbf{g}^{\prime \prime}+D_{\sigma}^{-1}\left(\varepsilon_{1} \mathbf{k}_{\mathbf{1}}^{\prime} \mathbf{g}-a \mathbf{k}_{2} \mathbf{l}\right)\right]\right\}
\end{aligned}
$$

where the derivative and antiderivative operators act on scalar fields as $\mathbf{f}^{\prime}(\gamma)=\mathbf{f}_{\gamma}^{\prime}$ and $D_{\sigma}^{-1}(\mathbf{f})(\gamma)=D_{\sigma}^{-1}\left(\mathbf{f}_{\gamma}\right)$, respectively. Thus $\mathfrak{X}_{\mathscr{A}}(\Lambda)$ stands for the set of $\mathscr{A}$-local vector fields locally preserving the pseudo arc-length parameter and the causal character. These vector fields commute with the tangent vector field $\mathbf{T}$, so they will be called evolution vector fields. We also denote by $\overline{\mathfrak{x}}_{\mathscr{A}}(\Lambda)$ and $\mathfrak{X}_{\mathscr{A}}^{*}(\Lambda)$ the sets of vector fields $\mathbf{V}$ such that $\mathbf{V}_{\gamma} \in \mathfrak{X}_{\mathscr{P}}(\gamma)$ and $\mathbf{V}_{\gamma} \in \mathfrak{X}_{\mathscr{P}}^{*}(\gamma)$, respectively. Hence,

$$
\begin{aligned}
& \overline{\mathfrak{X}}_{\mathscr{A}}(\Lambda) \\
& \quad=\left\{\mathbf{V}=\mathbf{f T}+\mathbf{h} \mathbf{W}_{\mathbf{1}}+\mathbf{g N}+\mathbf{l} \mathbf{W}_{\mathbf{2}}: \mathbf{f}, \mathbf{h}, \mathbf{g}, \mathbf{l} \in \mathscr{A}\right\} . \\
& \mathfrak{X}_{\mathscr{A}}^{*}(\Lambda)=\left\{\mathbf{V} \in \overline{\mathfrak{X}}_{\mathscr{A}}(\Lambda): \mathbf{h}=-\frac{\varepsilon_{1}}{a} \mathbf{g}^{\prime}\right\} .
\end{aligned}
$$

Remark 10. In what follows, we will operate with scalar fields and vector fields in the natural way, understanding that the result of the operation is again a scalar field or vector field. For instance, if $\mathbf{V}, \mathbf{U}$ are vector fields on $\Lambda$, then $\langle\mathbf{V}, \mathbf{U}\rangle$ is a scalar field, where $\langle\mathbf{V}, \mathbf{U}\rangle(\gamma)=\left\langle\mathbf{V}_{\gamma}, \mathbf{U}_{\gamma}\right\rangle$; or $\nabla_{\mathbf{T}} \mathbf{V}$ is again a vector field, where $\nabla_{\mathbf{T}} \mathbf{V}(\gamma)=\nabla_{\mathbf{T}_{\gamma}} \mathbf{V}_{\gamma}$ and so on.

Hence, for $\mathbf{V} \in \mathfrak{X}_{\mathscr{A}}^{*}(\Lambda)$, the operator $D_{\mathbf{V}}: \overline{\mathfrak{X}}_{\mathscr{A}}(\Lambda) \rightarrow$ $\overline{\mathfrak{X}}_{\mathscr{A}}(\Lambda)$ is defined as $\left(D_{\mathbf{V}} \mathbf{U}\right)(\gamma)=D_{\mathbf{V}_{\gamma}} \mathbf{U}_{\gamma}$. The operator $D_{\mathbf{V}}$ can also be described in other words when $\mathbf{V} \in \mathfrak{X}_{\mathscr{A}}(\Lambda)$. Consider $\gamma$ is a null curve in $\Lambda$ and suppose that $\mathbf{V}_{\gamma}(\sigma)=$ $(\partial \gamma / \partial t)(\sigma, 0)$, then

$$
\left(D_{\mathbf{V}} \mathbf{U}\right)_{\gamma}(\sigma)=\left.\frac{D}{\partial t}\right|_{t=0} \mathbf{U}_{\gamma_{t}}(\sigma) .
$$

In fact, the tensor derivation $D_{\mathrm{V}}$ is an extension of the Fréchet derivative defined in (15) for derivations to vector fields on the null curves space. In this way, this operator can be easily translated to the context of any other type of curves.

The Lie algebra structure on local vector fields locally preserving the causal character provided by Proposition 9 (along a particular curve) can also be easily extended on the set $\mathfrak{X}_{\mathscr{A}}^{*}(\Lambda)$.

Proposition 11. The map $[\cdot, \cdot]: \mathfrak{X}_{\mathscr{A}}^{*}(\Lambda) \times \mathfrak{X}_{\mathscr{A}}^{*}(\Lambda) \rightarrow \mathfrak{X}_{\mathscr{A}}^{*}(\Lambda)$ given by

$$
[\mathbf{V}, \mathbf{U}](\gamma)=\left[\mathbf{v}_{\gamma}, \mathbf{U}_{\gamma}\right]_{\gamma}
$$

is a Lie bracket verifying the following:

(a) $[\mathbf{V}, \mathbf{U}](\mathbf{f})=\mathbf{V U}(\mathbf{f})-\mathbf{U V}(\mathbf{f})$ for all $\mathbf{f} \in \mathscr{A}$.

(b) $[\cdot, \cdot]$ is closed for elements in $\mathfrak{X}_{\mathscr{A}}(\Lambda)$; that is, if $\mathbf{V}, \mathbf{U} \in$ $\mathfrak{X}_{\mathscr{A}}(\Lambda)$, then $[\mathbf{V}, \mathbf{U}] \in \mathfrak{X}_{\mathscr{A}}(\Lambda)$.

Hence, $[\cdot, \cdot]$ is a Lie bracket, $\left(\mathfrak{X}_{\mathscr{A}}^{*}(\Lambda),[\cdot, \cdot]\right)$ is a Lie algebra, and the space of evolution vector fields $\left(\mathfrak{X}_{\mathscr{A}}(\Lambda),[\cdot, \cdot]\right)$ is a Lie subalgebra of $\mathfrak{X}_{\mathscr{A}}^{*}(\Lambda)$.

Let us define $\operatorname{der}(\mathscr{A})$ as the set of derivations on $\mathscr{A}$ defined in the natural way and $\operatorname{der}^{*}(\mathscr{A})$ the Lie subalgebra of all evolution derivations. In this setting, the elements of $\operatorname{der}^{*}(\mathscr{A})$ are given by $\partial_{(\mathbf{p}, \mathbf{q})}$, with $\mathbf{p}, \mathbf{q} \in \mathscr{A}$, such that they are defined as usual by $\partial_{(\mathbf{p}, \mathbf{q})} \mathbf{f}(\gamma)=\partial_{\left(\mathbf{p}_{\gamma}, \mathbf{q}_{\gamma}\right)} \mathbf{f}_{\gamma}$, for all $\mathbf{f} \in \mathscr{A}$ and $\gamma \in \Lambda$. Each vector field $\mathbf{V}$ on $\Lambda$ can be regarded as a derivation on $\mathscr{A}$, acting on the generators $\mathbf{k}_{1}$ and $\mathbf{k}_{2}$ in the following way:

$$
\begin{aligned}
& \mathbf{V}\left(\mathbf{k}_{\mathbf{1}}\right)(\gamma)=\mathbf{V}_{\gamma}\left(\mathbf{k}_{\mathbf{1} \gamma}\right), \\
& \mathbf{V}\left(\mathbf{k}_{\mathbf{2}}\right)(\gamma)=\mathbf{V}_{\gamma}\left(\mathbf{k}_{\mathbf{2} \gamma}\right) .
\end{aligned}
$$

Theorem 12. The map $\Phi: \mathfrak{X}_{\mathscr{A}}(\Lambda) \rightarrow \operatorname{der}^{*}(\mathscr{A})$ defined by

$$
\Phi(\mathbf{V})=\partial_{\left(\mathbf{V}\left(\mathbf{k}_{1}\right), \mathbf{V}\left(\mathbf{k}_{2}\right)\right)}=\partial_{\Theta\left(\varphi_{\mathbf{V}},-\psi_{\mathbf{V}}\right)},
$$

where $\boldsymbol{\varphi}_{\mathbf{V}}=\varepsilon_{1}\left\langle\nabla_{\mathrm{T}} \mathbf{V}, \mathbf{W}_{\mathbf{1}}\right\rangle, \psi_{\mathbf{V}}=\varepsilon_{2}\left\langle\nabla_{\mathrm{T}} \mathbf{V}, \mathbf{W}_{2}\right\rangle$, and $\Theta$ is defined in (75), is a one-to-one homomorphism of Lie algebras. In particular, $\mathbf{V}_{1}$ and $\mathbf{V}_{2}$ are commuting vector fields with respect to the Lie bracket defined by Proposition 11 if and only if their corresponding curvature flows $\left(\mathbf{V}_{\mathbf{1}}\left(\mathbf{k}_{\mathbf{1}}\right), \mathbf{V}_{\mathbf{1}}\left(\mathbf{k}_{\mathbf{2}}\right)\right)$ and $\left(\mathbf{V}_{\mathbf{2}}\left(\mathbf{k}_{\mathbf{1}}\right), \mathbf{V}_{\mathbf{2}}\left(\mathbf{k}_{\mathbf{2}}\right)\right)$ commute with respect to the usual Lie bracket for scalar fields.

Proof. Since the map $\Phi$ is clearly linear, it is enough to prove that $\Phi$ keeps the Lie bracket, that is, $\Phi\left(\left[\mathbf{V}_{1}, \mathbf{V}_{2}\right]\right)=$ $\left[\Phi\left(\mathbf{V}_{1}\right), \Phi\left(\mathbf{V}_{2}\right)\right]$, the latter being equivalent to show that

$$
\begin{aligned}
& \partial_{\left(\left[\mathbf{V}_{1}, \mathbf{V}_{2}\right]\left(\mathbf{k}_{1}\right),\left[\mathbf{V}_{1}, \mathbf{V}_{2}\right]\left(\mathbf{k}_{2}\right)\right)} \\
& \quad=\left[\partial_{\left(\mathbf{V}_{\mathbf{1}}\left(\mathbf{k}_{1}\right), \mathbf{V}_{\mathbf{1}}\left(\mathbf{k}_{2}\right)\right)}, \partial_{\left(\mathbf{V}_{\mathbf{2}}\left(\mathbf{k}_{1}\right), \mathbf{V}_{2}\left(\mathbf{k}_{2}\right)\right)}\right] .
\end{aligned}
$$


From the last equality of Proposition 9(b) we deduce

$$
\begin{aligned}
& {\left[\mathbf{V}_{\mathbf{1} \gamma}, \mathbf{V}_{\mathbf{2} \gamma}\right]_{\gamma}\left(\mathbf{k}_{\mathbf{1} \gamma}\right)=\mathbf{V}_{\mathbf{1} \gamma} \mathbf{V}_{\mathbf{2} \gamma}\left(\mathbf{k}_{\mathbf{1} \gamma}\right)-\mathbf{V}_{\mathbf{2 \gamma}} \mathbf{V}_{\mathbf{1} \gamma}\left(\mathbf{k}_{\mathbf{1} \gamma}\right)} \\
& =\partial_{\left(\mathbf{V}_{\mathbf{1} \gamma}\left(\mathbf{k}_{\mathbf{1} \gamma}\right), \mathbf{V}_{\mathbf{1} \gamma}\left(\mathbf{k}_{\mathbf{2} \gamma}\right)\right)} \mathbf{V}_{\mathbf{2 \gamma}}\left(\mathbf{k}_{\mathbf{1} \gamma}\right) \\
& -\partial_{\left(\mathbf{V}_{2 \gamma}\left(\mathbf{k}_{1 \gamma}\right), \mathbf{V}_{2 \gamma}\left(\mathbf{k}_{2 \gamma}\right)\right)} \mathbf{V}_{\mathbf{1} \gamma}\left(\mathbf{k}_{\mathbf{1} \gamma}\right) \text {, } \\
& {\left[\mathbf{V}_{\mathbf{1} \gamma}, \mathbf{V}_{\mathbf{2 \gamma}}\right]_{\gamma}\left(\mathbf{k}_{\mathbf{2} \gamma}\right)=\mathbf{V}_{\mathbf{1} \gamma} \mathbf{V}_{\mathbf{2} \gamma}\left(\mathbf{k}_{2 \gamma}\right)-\mathbf{V}_{\mathbf{2} \gamma} \mathbf{V}_{\mathbf{1} \gamma}\left(\mathbf{k}_{\mathbf{2} \gamma}\right)} \\
& =\partial_{\left(\mathbf{V}_{\mathbf{1} \gamma}\left(\mathbf{k}_{\mathbf{1} \gamma}\right), \mathbf{V}_{\mathbf{1} \gamma}\left(\mathbf{k}_{2 \gamma}\right)\right)} \mathbf{V}_{2 \gamma}\left(\mathbf{k}_{\mathbf{2} \gamma}\right) \\
& -\partial_{\left(\mathbf{V}_{2 \gamma}\left(\mathbf{k}_{1 \gamma}\right), \mathbf{V}_{2 \gamma}\left(\mathbf{k}_{2 \gamma}\right)\right)} \mathbf{V}_{\mathbf{1} \gamma}\left(\mathbf{k}_{2 \gamma}\right) \text {, }
\end{aligned}
$$

and it is therefore satisfied

$$
\begin{aligned}
( & {\left.\left[\mathbf{V}_{\mathbf{1} \gamma}, \mathbf{V}_{\mathbf{2} \gamma}\right]_{\gamma}\left(\mathbf{k}_{\mathbf{1} \gamma}\right),\left[\mathbf{V}_{\mathbf{1} \gamma}, \mathbf{V}_{\mathbf{2} \gamma}\right]_{\gamma}\left(\mathbf{k}_{\mathbf{2} \gamma}\right)\right) } \\
= & \partial_{\left(\mathbf{V}_{\mathbf{1} \gamma}\left(\mathbf{k}_{\mathbf{1}}\right), \mathbf{V}_{\mathbf{1} \gamma}\left(\mathbf{k}_{\mathbf{2} \gamma}\right)\right)}\left(\mathbf{V}_{\mathbf{2} \gamma}\left(\mathbf{k}_{\mathbf{1} \gamma}\right), \mathbf{V}_{\mathbf{2} \gamma}\left(\mathbf{k}_{\mathbf{2} \gamma}\right)\right) \\
& -\partial_{\left.\left(\mathbf{V}_{2 \gamma}\left(\mathbf{k}_{\mathbf{1}}\right)\right) \mathbf{V}_{\mathbf{2} \gamma}\left(\mathbf{k}_{\mathbf{2} \gamma}\right)\right)}\left(\mathbf{V}_{\mathbf{1} \gamma}\left(\mathbf{k}_{\mathbf{1} \gamma}\right), \mathbf{V}_{\mathbf{1} \gamma}\left(\mathbf{k}_{\mathbf{2} \gamma}\right)\right) \\
= & {\left[\left(\mathbf{V}_{\mathbf{1} \gamma}\left(\mathbf{k}_{\mathbf{1} \gamma}\right), \mathbf{V}_{\mathbf{1} \gamma}\left(\mathbf{k}_{\mathbf{2} \gamma}\right)\right),\left(\mathbf{V}_{\mathbf{2} \gamma}\left(\mathbf{k}_{\mathbf{1} \gamma}\right), \mathbf{V}_{\mathbf{2} \gamma}\left(\mathbf{k}_{\mathbf{2} \gamma}\right)\right)\right] }
\end{aligned}
$$

for all $\gamma \in \Lambda$. Finally, formula (83) is followed from

$$
\begin{aligned}
& {\left[\partial_{\left(\mathbf{V}_{1 \gamma}\left(\mathbf{k}_{1 \gamma}\right), \mathbf{V}_{\mathbf{1}}\left(\mathbf{k}_{2 \gamma}\right)\right)}, \partial_{\left(\mathbf{V}_{2 \gamma}\left(\mathbf{k}_{1 \gamma}\right), \mathbf{V}_{2 \gamma}\left(\mathbf{k}_{2 \gamma}\right)\right)}\right]} \\
& \quad=\partial_{\left[\left(\mathbf{V}_{1 \gamma}\left(\mathbf{k}_{1 \gamma}\right), \mathbf{V}_{1 \gamma}\left(\mathbf{k}_{2 \gamma}\right)\right),\left(\mathbf{V}_{2 \gamma}\left(\mathbf{k}_{1 \gamma}\right), \mathbf{V}_{2 \gamma}\left(\mathbf{k}_{2 \gamma}\right)\right)\right]}
\end{aligned}
$$

In order to prove the injectivity we will prove that $\partial_{\left(\mathbf{V}\left(\mathbf{k}_{1}\right), \mathbf{V}\left(\mathbf{k}_{2}\right)\right)}=0$ implies $\mathbf{V}=0$, which is equivalent to proving that $\mathbf{V}\left(\mathbf{k}_{\mathbf{1}}\right)=\mathbf{V}\left(\mathbf{k}_{\mathbf{2}}\right)=0$ implies $\mathbf{V}=0$. As a first step we will prove that if $\mathbf{V}\left(\mathbf{k}_{\mathbf{1}}\right)=\mathbf{V}\left(\mathbf{k}_{\mathbf{2}}\right)=0$, then $\varphi_{\mathbf{V}}=\psi_{\mathbf{V}}=0$ and so, from formula (46), $\nabla_{\mathrm{T}} \mathbf{V}=0$. According to (74) we have that

$$
\begin{gathered}
\left(\mathbf{V}\left(\mathbf{k}_{\mathbf{1}}\right), \mathbf{V}\left(\mathbf{k}_{\mathbf{2}}\right)\right)=\Theta\left(\boldsymbol{\varphi}_{\mathrm{V}},-\psi_{\mathbf{V}}\right)=\frac{1}{a}\left(\theta\left(\mathbf{k}_{\mathbf{1}}\right) \boldsymbol{\varphi}_{\mathrm{V}}\right. \\
\left.-S\left(\mathbf{k}_{\mathbf{2}}\right) \psi_{\mathbf{V}}, S\left(\mathbf{k}_{\mathbf{2}}\right) \boldsymbol{\varphi}_{\mathrm{V}}+\varepsilon_{1} \varepsilon_{2} \theta\left(\mathbf{k}_{\mathbf{1}}\right) \psi_{\mathrm{V}}\right) .
\end{gathered}
$$

For a scalar field $\mathbf{f} \in \mathscr{A}$ we denote by ord(f) the order of the highest derivative (with respect to both $\mathbf{k}_{\mathbf{1}}$ or $\mathbf{k}_{\mathbf{2}}$ ) appearing in $\mathbf{f}$; that is,

$$
\operatorname{ord}(\mathbf{f})=\max \left\{i: \frac{\partial \mathbf{f}}{\partial \mathbf{k}_{\mathbf{1}}^{(i)}} \neq 0 \text { or } \frac{\partial \mathbf{f}}{\partial \mathbf{k}_{\mathbf{2}}^{(i)}} \neq 0\right\} \text {. }
$$

Suppose that $\operatorname{ord}\left(\varphi_{\mathrm{V}}\right)=n \neq 0$; then we have that $\operatorname{ord}\left(\theta\left(\mathbf{k}_{\mathbf{1}}\right) \boldsymbol{\varphi}_{\mathbf{V}}\right)=n+3$. Since $\mathbf{V}\left(\mathbf{k}_{\mathbf{1}}\right)=0$ it is necessarily obtained that $\operatorname{ord}\left(S\left(\mathbf{k}_{\mathbf{2}}\right) \psi_{\mathbf{V}}\right)=n+3$, whence ord $\left(\psi_{\mathbf{V}}\right)=n+2$. Accordingly, ord $\left(\theta\left(\mathbf{k}_{\mathbf{1}}\right) \psi_{\mathbf{V}}\right)=n+5$, which together with the equation $\mathbf{V}\left(\mathbf{k}_{2}\right)=0$ would lead to $\operatorname{ord}\left(\varphi_{\mathrm{V}}\right)=n+4$ and so a contradiction. Therefore the scalar field $\varphi_{\mathrm{V}}$ is constant, $\varphi_{\mathrm{V}}=c$, and it would verify the equation

$$
0=\frac{1}{a}\left(\theta\left(\mathbf{k}_{\mathbf{1}}\right) \boldsymbol{\varphi}_{\mathrm{V}}-S\left(\mathbf{k}_{\mathbf{2}}\right) \psi\right)=\frac{1}{a}\left(c \mathbf{k}_{\mathbf{1}}^{\prime}-S\left(\mathbf{k}_{\mathbf{2}}\right) \psi_{\mathbf{V}}\right) .
$$

The latter equation is satisfied if and only if $c=0$ and $\psi_{\mathrm{V}}=0$, that is, $\nabla_{\mathbf{T}} \mathbf{V}=0$. Using formula (37), the equation $\nabla_{\mathbf{T}} \mathbf{V}=0$ can be developed as

$$
\begin{aligned}
\mathbf{f}_{\mathbf{V}}^{\prime}-\mathbf{k}_{\mathbf{1}} \mathbf{h}_{\mathbf{V}}+\mathbf{k}_{\mathbf{2}} \mathbf{l}_{\mathbf{V}}=0 \\
a \mathbf{f}_{\mathbf{V}}+\mathbf{h}_{\mathbf{V}}^{\prime}-\varepsilon_{1} \mathbf{k}_{\mathbf{1}} \mathbf{g}_{\mathbf{V}}=0 \\
\varepsilon_{1} a \mathbf{h}_{\mathbf{V}}+\mathbf{g}_{\mathrm{V}}^{\prime}=0 \\
\mathbf{l}_{\mathbf{V}}^{\prime}+\varepsilon_{2} \mathbf{k}_{2} \mathbf{g}_{\mathbf{V}}=0 .
\end{aligned}
$$

Suppose that $\operatorname{ord}\left(\mathbf{l}_{\mathrm{V}}\right)=n \neq 0$; then (90) give rise to the following implications:

$$
\begin{aligned}
& \operatorname{ord}\left(\mathbf{g}_{\mathbf{V}}\right)=n+1 \Longrightarrow \\
& \operatorname{ord}\left(\mathbf{h}_{\mathbf{V}}\right)=n+2 \Longrightarrow \\
& \operatorname{ord}\left(\mathbf{f}_{\mathbf{V}}\right)=n+3 .
\end{aligned}
$$

Nevertheless, those orders of derivation represent a direct contradiction to the first equation in (90) unless $\mathbf{l}=\mathbf{g}=\mathbf{h}=$ $\mathbf{f}=0$.

Remark 13. From Theorem 12 we have that $\operatorname{Im}(\Phi)$ is a Lie subalgebra of the algebra $\operatorname{der}^{*}(\mathscr{A})$ of all evolution derivations. Thus, we conclude that the algebra of evolution vector fields $\mathfrak{X}_{\mathscr{A}}(\Lambda)$ on $\Lambda$ can be regarded as a Lie subalgebra of the evolution derivations.

Consider the vector fields $\mathbf{V}_{\mathbf{0}}=b \mathbf{T}$ and $\mathbf{V}_{1}=-a c \mathbf{k}_{\mathbf{1}} \mathbf{T}-$ $2 \varepsilon_{1} a^{2} c \mathbf{N}$ borrowed from Example 8. Their flows $\gamma_{t} \in \Lambda$ are governed by

$$
\begin{aligned}
& \frac{d}{d t}\left(\gamma_{t}\right)=\mathbf{V}_{\mathbf{0} \gamma_{t}}=b \mathbf{T}_{\gamma_{t}}, \\
& \frac{d}{d t}\left(\gamma_{t}\right)=\mathbf{V}_{\mathbf{1} \gamma_{t}}=-a c \mathbf{k}_{\mathbf{1} \gamma_{t}} \mathbf{T}_{\gamma_{t}}-2 \varepsilon_{1} a^{2} c \mathbf{N}_{\gamma_{t}},
\end{aligned}
$$

which in turn induce evolutions for the curvature functions $\mathbf{k}_{\mathbf{1}}$ and $\mathbf{k}_{\mathbf{2}}$ given by

$$
\begin{aligned}
\frac{d}{d t}\left(\mathbf{k}_{\mathbf{1} \gamma_{t}}\right) & =\mathbf{V}_{\mathbf{0} \gamma_{t}}\left(\mathbf{k}_{\mathbf{1} \gamma_{t}}\right)=b \mathbf{k}_{\mathbf{1} \gamma_{t}}^{\prime}, \\
\frac{d}{d t}\left(\mathbf{k}_{\mathbf{2} \gamma_{t}}\right) & =\mathbf{V}_{\mathbf{0} \gamma_{t}}\left(\mathbf{k}_{\mathbf{2} \gamma_{t}}\right)=b \mathbf{k}_{\mathbf{2} \gamma_{t}}^{\prime}, \\
\frac{d}{d t}\left(\mathbf{k}_{\mathbf{1} \gamma_{t}}\right) & =\mathbf{V}_{\mathbf{1} \gamma_{t}}\left(\mathbf{k}_{\mathbf{1} \gamma_{t}}\right) \\
& =c\left(\mathbf{k}_{\mathbf{1} \gamma_{t}}^{(3)}+3 a \mathbf{k}_{\mathbf{1} \gamma_{t}} \mathbf{k}_{\mathbf{1} \gamma_{t}}^{\prime}+6 \varepsilon_{1} \varepsilon_{2} a \mathbf{k}_{\mathbf{2} \gamma_{t}} \mathbf{k}_{\mathbf{2} \gamma_{t}}^{\prime}\right), \\
\frac{d}{d t}\left(\mathbf{k}_{\mathbf{2} \gamma_{t}}\right) & =\mathbf{V}_{\mathbf{1} \gamma_{t}}\left(\mathbf{k}_{\mathbf{2} \gamma_{t}}\right)=-c\left(2 \mathbf{k}_{\mathbf{2} \gamma_{t}}^{(3)}+3 a \mathbf{k}_{\mathbf{1} \gamma_{t}} \mathbf{k}_{\mathbf{2} \gamma_{t}}^{\prime}\right) .
\end{aligned}
$$

Observe that the evolution equation (95) is the HirotaSatsuma equation (1) (up to scaling the variables $t, x$ and rescaling the functions $u, v)$. Besides, the flows associated to the curvatures given by $\mathbf{V}_{\mathbf{0}}$ and $\mathbf{V}_{\mathbf{1}}$ are basically flows $\sigma_{0}$ and $\sigma_{1}$ given in (3). We refer to (93) induced by $\mathbf{V}_{\mathbf{1}}$ (which also 
appears in [3]) as the null localized induction equation (NLIE). Theorem 12 will be used below to obtain a recursion operator for NLIE and thereby prove its integrability.

Proposition 14. The operator $\mathbf{R}$ acting on symmetries $\mathbf{V}$,

$$
\mathbf{R}(\mathbf{V})=\mathscr{X}\left(\frac{1}{2} \mathbf{V}\left(\mathbf{k}_{\mathbf{1}}\right),-\varepsilon_{1} \varepsilon_{2} \mathbf{V}\left(\mathbf{k}_{\mathbf{2}}\right)\right)
$$

is a recursion operator for NLIE.

Proof. Let $\mathbf{U}=\mathbf{R}(\mathbf{V})$. Then by definition and making use of (76) we obtain

$$
\begin{aligned}
\left(\mathbf{U}\left(\mathbf{k}_{\mathbf{1}}\right), \mathbf{U}\left(\mathbf{k}_{\mathbf{2}}\right)\right) & =R\left(2 \mathbf{h}_{\mathbf{U}},-\varepsilon_{1} \varepsilon_{2} \mathbf{l}_{\mathbf{U}}\right) \\
& =R\left(\mathbf{V}\left(\mathbf{k}_{\mathbf{1}}\right), \mathbf{V}\left(\mathbf{k}_{\mathbf{2}}\right)\right) .
\end{aligned}
$$

The result can be easily deduced as a consequence of Theorem 12 .

We now proceed with the construction of an infinite hierarchy of symmetries following the same scheme as in (3):

$$
\begin{aligned}
\mathbf{V}_{\mathbf{2 n}} & =\mathbf{R}^{n} \mathbf{V}_{\mathbf{0}} ; \\
\mathbf{V}_{\mathbf{2 n + 1}} & =\mathbf{R}^{n} \mathbf{V}_{\mathbf{1}} .
\end{aligned}
$$

Then, we have

$$
\begin{aligned}
\mathbf{V}_{\mathbf{2}}= & \mathbf{R} \mathbf{V}_{\mathbf{0}} \\
= & \left(-\frac{b}{4 a} \mathbf{k}_{\mathbf{1}}^{\prime \prime}-\frac{b}{8} \mathbf{k}_{\mathbf{1}}^{2}+\frac{\varepsilon_{1} \varepsilon_{2} b}{4} \mathbf{k}_{2}^{2}+\frac{\varepsilon_{1} c_{1}}{2 a} \mathbf{k}_{\mathbf{1}}+c_{2}\right) \mathbf{T} \\
& +\frac{b}{2} \mathbf{k}_{\mathbf{1}}^{\prime} \mathbf{W}_{\mathbf{1}}+\left(c_{1}-\frac{\varepsilon_{1} a b}{2} \mathbf{k}_{\mathbf{1}}\right) \mathbf{N}-\varepsilon_{1} \varepsilon_{2} b \mathbf{k}_{\mathbf{2}}^{\prime} \mathbf{W}_{\mathbf{2}}
\end{aligned}
$$

and the corresponding curvature flow is

$$
\begin{aligned}
& \mathbf{V}_{\mathbf{2}}\left(\mathbf{k}_{\mathbf{1}}\right)=\frac{1}{8 a^{2}}\left[2 b \mathbf{k}_{\mathbf{1}}^{(5)}+\left(10 a b \mathbf{k}_{\mathbf{1}}-4 \varepsilon_{1} c_{1}\right) \mathbf{k}_{\mathbf{1}}^{(3)}\right. \\
& +20 \varepsilon_{1} \varepsilon_{2} a b \mathbf{k}_{\mathbf{2}} \mathbf{k}_{\mathbf{2}}^{(3)}+20 a b \mathbf{k}_{\mathbf{1}}^{\prime} \mathbf{k}_{\mathbf{1}}^{\prime \prime}+20 \varepsilon_{1} \varepsilon_{2} a b \mathbf{k}_{\mathbf{2}}^{\prime} \mathbf{k}_{2}^{\prime \prime} \\
& +\left(15 a^{2} b \mathbf{k}_{\mathbf{1}}^{2}+10 \varepsilon_{1} \varepsilon_{2} a^{2} b \mathbf{k}_{2}^{2}-12 \varepsilon_{1} a c_{1} \mathbf{k}_{\mathbf{1}}+8 a^{2} c_{2}\right) \\
& \left.\cdot \mathbf{k}_{\mathbf{1}}^{\prime}+\left(20 \varepsilon_{1} \varepsilon_{2} a^{2} b \mathbf{k}_{\mathbf{1}}-24 \varepsilon_{2} a c_{1}\right) \mathbf{k}_{\mathbf{2}} \mathbf{k}_{\mathbf{2}}^{\prime}\right] \\
& \mathbf{V}_{\mathbf{2}}\left(\mathbf{k}_{\mathbf{2}}\right)=\frac{1}{8 a^{2}}\left[-8 b \mathbf{k}_{\mathbf{2}}^{(5)}+\left(8 \varepsilon_{1} c_{1}-20 a b \mathbf{k}_{\mathbf{1}}\right) \mathbf{k}_{\mathbf{2}}^{(3)}\right. \\
& \quad-10 a b \mathbf{k}_{\mathbf{1}}^{\prime \prime} \mathbf{k}_{\mathbf{2}}^{\prime}-20 a b \mathbf{k}_{\mathbf{1}}^{\prime} \mathbf{k}_{2}^{\prime \prime} \\
& +\left(10 \varepsilon_{1} \varepsilon_{2} a^{2} b \mathbf{k}_{2}^{2}-5 a^{2} b \mathbf{k}_{\mathbf{1}}^{2}+12 \varepsilon_{1} a c_{1} \mathbf{k}_{\mathbf{1}}+8 a^{2} c_{2}\right) \\
& \left.\quad \cdot \mathbf{k}_{2}^{\prime}\right]
\end{aligned}
$$

Likewise, the next vector field in the hierarchy becomes

$$
\begin{aligned}
\mathbf{V}_{\mathbf{3}} & =\mathbf{R V}_{\mathbf{1}}=\left(-\frac{c}{4 a} \mathbf{k}_{\mathbf{1}}^{(4)}-\frac{3 c}{4} \mathbf{k}_{\mathbf{1}} \mathbf{k}_{\mathbf{1}}^{\prime \prime}-\frac{7 c}{8}\left(\mathbf{k}_{\mathbf{1}}^{\prime}\right)^{2}\right. \\
& -\frac{5 \varepsilon_{1} \varepsilon_{2} c}{2} \mathbf{k}_{\mathbf{2}} \mathbf{k}_{\mathbf{2}}^{\prime \prime}-\varepsilon_{1} \varepsilon_{2} c\left(\mathbf{k}_{2}^{\prime}\right)^{2}-\frac{a c}{8} \mathbf{k}_{\mathbf{1}}^{3}+\frac{\varepsilon_{1} c_{3}}{2 a} \mathbf{k}_{\mathbf{1}} \\
& \left.-\frac{3 \varepsilon_{1} \varepsilon_{2} a c}{4} \mathbf{k}_{\mathbf{1}} \mathbf{k}_{2}^{2}+c_{4}\right) \mathbf{T}+\left(\frac{c}{2} \mathbf{k}_{\mathbf{1}}^{(3)}+\frac{3 a c}{2} \mathbf{k}_{\mathbf{1}} \mathbf{k}_{\mathbf{1}}^{\prime}\right. \\
& \left.+3 \varepsilon_{1} \varepsilon_{2} a c \mathbf{k}_{\mathbf{2}} \mathbf{k}_{\mathbf{2}}^{\prime}\right) \mathbf{W}_{\mathbf{1}}+\left(-\frac{\varepsilon_{1} a c}{2} \mathbf{k}_{\mathbf{1}}^{\prime \prime}-\frac{3 \varepsilon_{1} a^{2} c}{4} \mathbf{k}_{\mathbf{1}}^{2}\right. \\
& \left.-\frac{3 \varepsilon_{2} a^{2} c}{2} \mathbf{k}_{\mathbf{2}}^{2}+c_{3}\right) \mathbf{N}+\left(2 \varepsilon_{1} \varepsilon_{2} c \mathbf{k}_{\mathbf{2}}^{(3)}+3 \varepsilon_{1} \varepsilon_{2} a c \mathbf{k}_{\mathbf{1}} \mathbf{k}_{\mathbf{2}}^{\prime}\right) \\
& \cdot \mathbf{W}_{\mathbf{2}} .
\end{aligned}
$$

Note that the above geometric hierarchy of commuting vector fields at the curve level is a generalization of the ones obtained in [1] for the 3-dimensional case, albeit using a different procedure. In fact, it was not possible to extend the procedure used in [1] to obtain the recursion operator and the Hamiltonian structure at the curve level to the 4-dimensional setting, mainly because of the appearance of nonlocal vector fields. Searching for a Hamiltonian structure at the curve level for the 4-dimensional case will be one of the subject for future research.

\section{Conclusions}

In this paper, our primary aim was to study the integrability properties of null curve evolutions in a flat 4-dimensional background. We undertook our research in an enough degree of generality for the purpose of showing the role of the constants appearing on it, especially when they possess geometrical meaning. In that regard, the way in which the computations were conducted to expose the most important elements of the Hamiltonian structure for curvature flows is particularly important. One of the most surprising facts was to obtain the recursion operator (split into both the Poisson operator and the symplectic operator in formulas (74) and (75)) of the Hirota-Satsuma system by means of the geometry of null curves or, more precisely, making use of the projection of convenient variation vector fields onto the screen bundle. Similar results were obtain in $[19,20]$, suggesting that the screen bundle of a null curve may be thought of as playing the same role of the normal bundle in a Riemannian curve. We can therefore also state the following important conclusion: if we have a evolution vector field $\mathbf{V}$ with $\left(\varphi_{\mathrm{V}}, \psi_{\mathrm{V}}\right)$ having the property of being the gradient of a certain functional $\mathbf{H}$, then the flow associated to the curvatures $\left(\mathbf{V}\left(\mathbf{k}_{\mathbf{1}}\right), \mathbf{V}\left(\mathbf{k}_{\mathbf{2}}\right)\right)$ is a completely integrable Hamiltonian system.

Furthermore, in Proposition 14 we have lifted the recursion operator for the Hirota-Satsuma system (at the curvature level) to a recursion operator for the NLIE equation (at the curve level), enabling us to obtain an infinite hierarchy of commuting vector fields. Proposition 11 shows that the subspace consisting of $\mathscr{A}$-local evolution vector fields 
(denoted by $\mathfrak{X}_{\mathscr{A}}(\Lambda)$ ) is closed under bracket and contains the commuting flows as a subalgebra.

One of the many benefits of increasing the dimension of the ambient space has been that the connections between integrable hierarchies of both null curves and their curvature flows become clearer. Nevertheless, finding a Hamiltonian structure at the curve level still needs to be achieved. In addition, it would be interesting to develop a purely geometric method to construct the existing structures of the dynamic of null curve motions without lifting any element from the curvature flow. Accordingly, further work is needed, perhaps in a nonlocal background, if possible, to properly understand which also has appeared in different contexts.

\section{Competing Interests}

The authors declare that there is no conflict of interests regarding the publication of this paper.

\section{Acknowledgments}

This work has been partially supported by MINECO-FEDER (Ministerio de Economía y Competitividad-FEDER) Project MTM2015-65430-P and Fundación Séneca (Región de Murcia) Project 19901/GERM/15, Spain.

\section{References}

[1] J. Del Amor, A. Giménez, and P. Lucas, "Hamiltonian structure for null curve evolution," Nonlinearity, vol. 27, no. 11, pp. 26272641, 2014.

[2] E. Musso and L. Nicolodi, "Hamiltonian flows on null curves," Nonlinearity, vol. 23, no. 9, pp. 2117-2129, 2010.

[3] Y.-Y. Li, "Motions of Cartan curves in $n$-dimensional Minkowski space," Modern Physics Letters A, vol. 28, no. 27, Article ID 1350110, 13 pages, 2013.

[4] R. Hirota and J. Satsuma, "Soliton solutions of a coupled Korteweg-de Vries equation," Physics Letters A, vol. 85, no. 8-9, pp. 407-408, 1981.

[5] R. Dodd and A. Fordy, "On the integrability of a system of coupled KdV equations," Physics Letters A, vol. 89, no. 4, pp. 168-170, 1982.

[6] J. Weiss, "Modified equations, rational solutions, and the Painlevé property for the Kadomtsev-PETviashvili and HirotaSatsuma equations," Journal of Mathematical Physics, vol. 26, no. 9, pp. 2174-2180, 1985.

[7] J. Weiss, "The sine-Gordon equations: complete and partial integrability," Journal of Mathematical Physics, vol. 25, no. 7, pp. 2226-2235, 1984.

[8] D. Levi, "A hierarchy of coupled Korteweg-de Vries equations," Physics Letters. A, vol. 95, no. 1, pp. 7-10, 1983.

[9] S. B. Leble and N. V. Ustinov, "Darboux transforms, deep reductions and solitons," Journal of Physics A: Mathematical and General, vol. 26, no. 19, pp. 5007-5016, 1993.

[10] H. C. Hu and Q. P. Liu, "New Darboux transformation for Hirota-Satsuma coupled KdV system," Chaos, Solitons and Fractals, vol. 17, no. 5, pp. 921-928, 2003.

[11] H. C. Hu and Y. Liu, "New positon, negaton and complexiton solutions for the Hirota-Satsuma coupled KdV system," Physics
Letters, Section A: General, Atomic and Solid State Physics, vol. 372, no. 36, pp. 5795-5798, 2008.

[12] B. Fuchssteiner, "The Lie algebra structure of degenerate Hamiltonian and bi-Hamiltonian systems," Progress of Theoretical Physics, vol. 68, no. 4, pp. 1082-1104, 1982.

[13] W. Oevel, "On the integrability of the Hirota-Satsuma system," Physics Letters A, vol. 94, no. 9, pp. 404-407, 1983.

[14] A. Nersessian and E. Ramos, "Massive spinning particles and the geometry of null curves," Physics Letters, Section B: Nuclear, Elementary Particle and High-Energy Physics, vol. 445, no. 1-2, pp. 123-128, 1998.

[15] A. Nersessian, R. Manvelyan, and H. J. Müller-Kirsten, "Particle with torsion on 3d null-curves," Nuclear Physics B: Proceedings Supplement, vol. 88, no. 1-3, pp. 381-384, 2000.

[16] A. Ferrández, A. Giménez, and P. Lucas, "Geometrical particle models on 3D null curves," Physics Letters B, vol. 543, no. 3-4, pp. 311-317, 2002.

[17] A. Ferrández, A. Giménez, and P. Lucas, "Relativistic particles and the geometry of 4-D null curves," Journal of Geometry and Physics, vol. 57, no. 10, pp. 2124-2135, 2007.

[18] A. Giménez, "Relativistic particles along null curves in 3D Lorentzian space forms," International Journal of Bifurcation and Chaos in Applied Sciences and Engineering, vol. 20, no. 9, pp. 2851-2859, 2010.

[19] G. Marí Beffa, J. A. Sanders, and J. P. Wang, "Integrable systems in three-dimensional Riemannian geometry," Journal of Nonlinear Science, vol. 12, no. 2, pp. 143-167, 2002.

[20] J. A. Sanders and J. P. Wang, "Integrable systems in $n$ dimensional Riemannian geometry," Moscow Mathematical Journal, vol. 3, no. 4, pp. 1369-1393, 2003.

[21] J. Langer and R. Perline, "Poisson geometry of the filament equation," Journal of Nonlinear Science, vol. 1, no. 1, pp. 71-93, 1991.

[22] E. L. Mansfield and P. H. van der Kamp, "Evolution of curvature invariants and lifting integrability," Journal of Geometry and Physics, vol. 56, no. 8, pp. 1294-1325, 2006.

[23] J. Del Amor, A. Giménez, and P. Lucas, "A Lie algebra structure on variation vector fields along curves in 2-dimensional space forms," Journal of Geometry and Physics, vol. 88, pp. 94-104, 2015.

[24] I. Dorfman, Dirac Structures and Integrability of Nonlinear Evolution Equations, John Wiley \& Sons, Chichester, UK, 1993.

[25] L. A. Dickey, Soliton Equations and Hamiltonian Systems, vol. 26 of Advanced Series in Mathematical Physics, World Scientific, River Edge, NJ, USA, 2nd edition, 2003.

[26] M. Blaszak, Multi-Hamiltonian Theory of Dynamical Systems, Springer Science \& Business Media, 2012.

[27] A. V. Mikhailov, A. B. Shabat, and R. I. Yamilov, “The symmetry approach to the classification of non-linear equations. Complete lists of integrable systems," Russian Mathematical Surveys, vol. 42, no. 4, pp. 1-63, 1987.

[28] V. V. Sokolov, "Symmetries of evolution equations," Russian Mathematical Surveys, vol. 43, no. 5, pp. 165-204, 1988.

[29] A. V. Mikhailov, A. B. Shabat, and V. V. Sokolov, "The symmetry approach to classification of integrable equations," in What Is Integrability? V. E. Zakharov, Ed., Springer Series in Nonlinear Dynamics, pp. 115-184, Springer, Berlin, Germany, 1991.

[30] A. V. Mikhailov and R. I. Yamilov, "Towards classification of (2+ 1)-dimensional integrable equations. Integrability conditions. I," Journal of Physics A: Mathematical and General, vol. 31, no. 31, pp. 6707-6715, 1998. 
[31] A. Ferrández, A. Giménez, and P. Lucas, "Null helices in Lorentzian space forms," International Journal of Modern Physics A, vol. 16, no. 30, pp. 4845-4863, 2001.

[32] Y. Yasui and N. Sasaki, "Differential geometry of the vortex filament equation," Journal of Geometry and Physics, vol. 28, no. 1-2, pp. 195-207, 1998. 


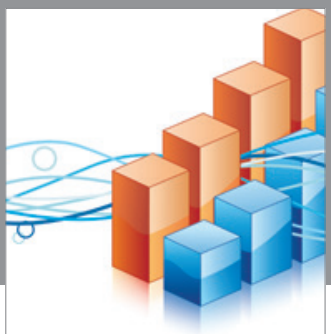

Advances in

Operations Research

vatem alat4

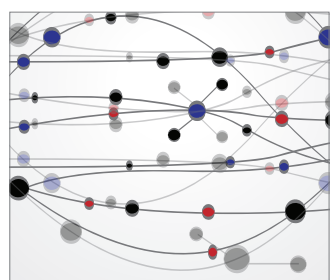

\section{The Scientific} World Journal
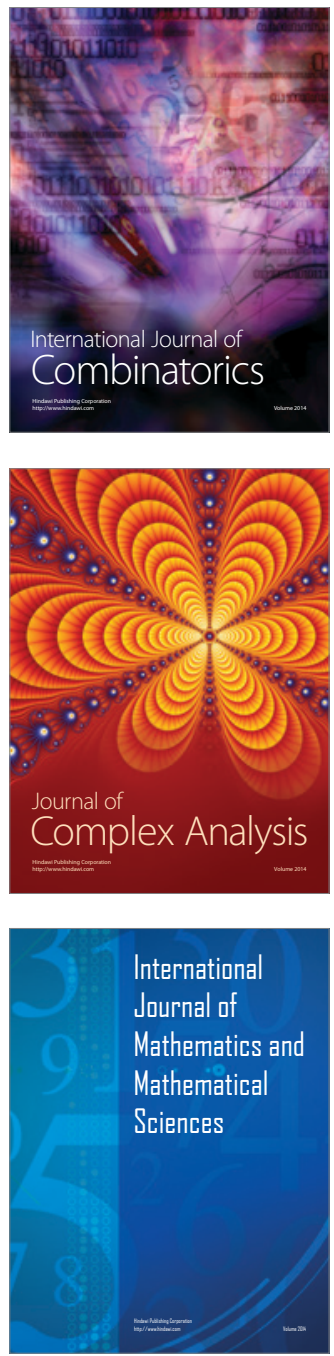
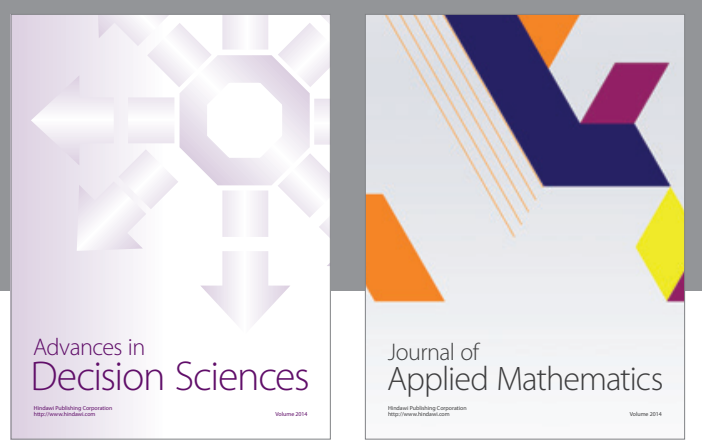

Algebra

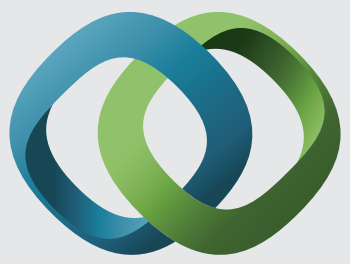

\section{Hindawi}

Submit your manuscripts at

http://www.hindawi.com
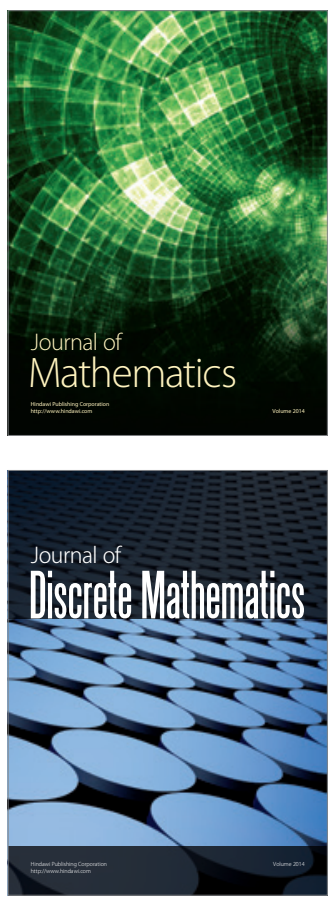

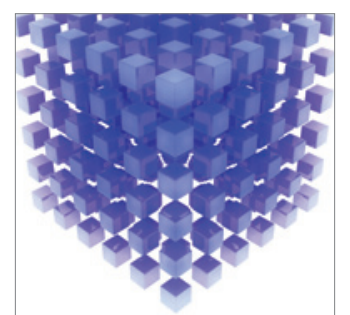

Mathematical Problems in Engineering
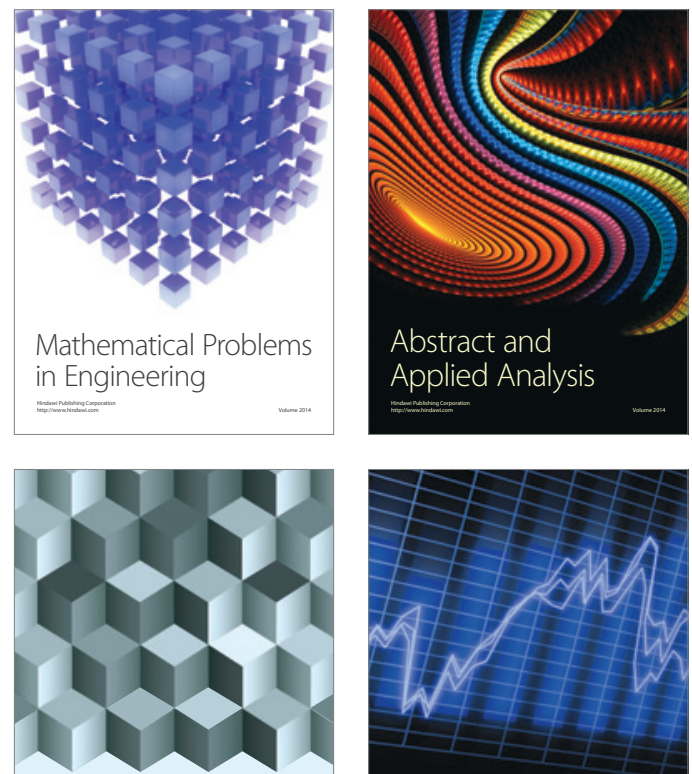

Journal of

Function Spaces

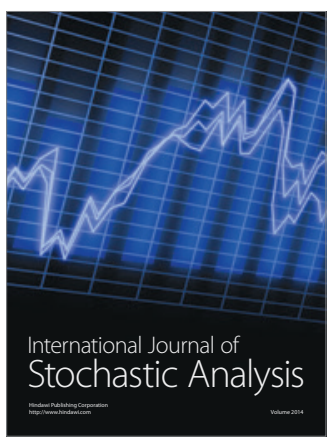

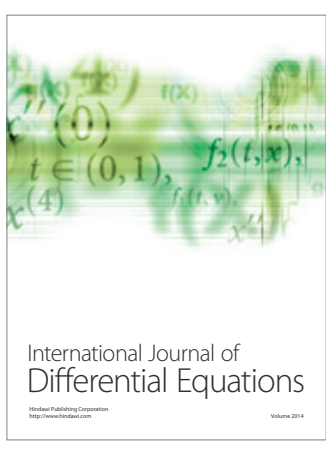
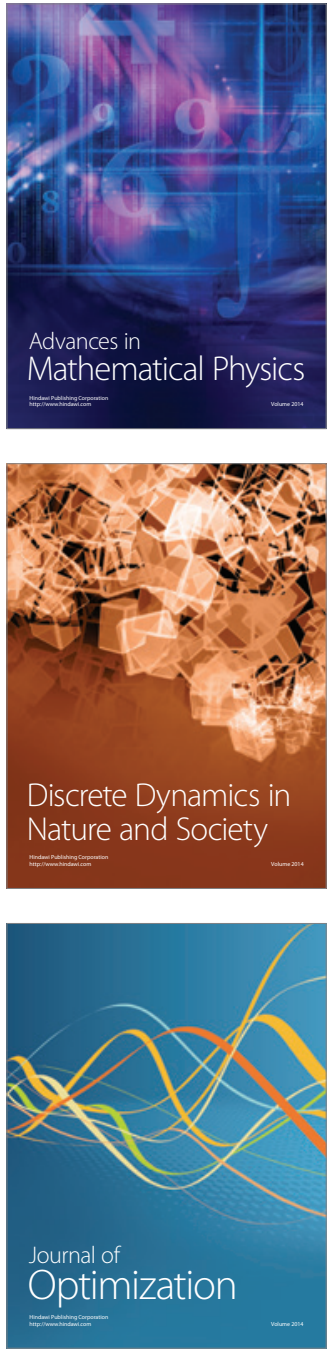